\title{
ON THE NATURE OF THE DEEPLY EMBEDDED PROTOSTAR OMC-2 FIR 4
}

\author{
E. Furlan ${ }^{1,10}$, S. T. Megeath ${ }^{2}$, M. Osorio ${ }^{3}$, A. M. Stutz ${ }^{4}$, W. J. Fischer ${ }^{2,11}$, B. Ali ${ }^{5}$, \\ T. STANKE ${ }^{6}$, P. MANOJ ${ }^{7}$, J. D. AdAMS ${ }^{8}$, AND J. J. TOBIN ${ }^{9,12}$ \\ ${ }^{1}$ National Optical Astronomy Observatory, 950 N. Cherry Avenue, Tucson, AZ 85719, USA; furlan@ipac.caltech.edu \\ ${ }^{2}$ Ritter Astrophysical Observatory, Department of Physics and Astronomy, University of Toledo, 2801 W. Bancroft Street, Toledo, OH 43606, USA \\ ${ }^{3}$ Instituto de Astrofísica de Andalucía, CSIC, Camino Bajo de Huétor 50, E-18008 Granada, Spain \\ ${ }^{4}$ Max-Planck-Institut für Astronomie, Königstuhl 17, D-69117 Heidelberg, Germany \\ ${ }^{5}$ NHSC/IPAC, California Institute of Technology, 770 S. Wilson Ave., Pasadena, CA 91125, USA \\ ${ }^{6}$ ESO, Karl-Schwarzschild-Strasse 2, D-85748, Garching bei München, Germany \\ ${ }^{7}$ Department of Astronomy and Astrophysics, Tata Institute of Fundamental Research, Homi Bhabha Road, Colaba, Mumbai 400005, India \\ ${ }^{8}$ USRA-SOFIA, DAOF, 2825 E. Ave. P, Palmdale, CA 93550, USA \\ ${ }^{9}$ National Radio Astronomy Observatory, Charlotttesville, VA 22903, USA \\ Received 2014 February 2; accepted 2014 March 10; published 2014 April 14
}

\begin{abstract}
We use mid-infrared to submillimeter data from the Spitzer, Herschel, and Atacama Pathfinder Experiment telescopes to study the bright submillimeter source OMC-2 FIR 4. We find a point source at 8, 24, and 70 $\mu \mathrm{m}$, and a compact, but extended source at 160,350 , and $870 \mu \mathrm{m}$. The peak of the emission from 8 to $70 \mu \mathrm{m}$, attributed to the protostar associated with FIR 4, is displaced relative to the peak of the extended emission; the latter represents the large molecular core the protostar is embedded within. We determine that the protostar has a bolometric luminosity of $37 L_{\odot}$, although including more extended emission surrounding the point source raises this value to $86 L_{\odot}$. Radiative transfer models of the protostellar system fit the observed spectral energy distribution well and yield a total luminosity of most likely less than $100 L_{\odot}$. Our models suggest that the bolometric luminosity of the protostar could be as low as 12-14 $L_{\odot}$, while the luminosity of the colder $(\sim 20 \mathrm{~K})$ extended core could be around $100 L_{\odot}$, with a mass of about $27 M_{\odot}$. Our derived luminosities for the protostar OMC-2 FIR 4 are in direct contradiction with previous claims of a total luminosity of $1000 L_{\odot}$. Furthermore, we find evidence from far-infrared molecular spectra and $3.6 \mathrm{~cm}$ emission that FIR 4 drives an outflow. The final stellar mass the protostar will ultimately achieve is uncertain due to its association with the large reservoir of mass found in the cold core.
\end{abstract}

Key words: circumstellar matter - infrared: stars - stars: formation - stars: individual (OMC-2 FIR 4) stars: protostars

Online-only material: color figures

\section{INTRODUCTION}

The OMC 2 region in the Orion A molecular cloud is actively forming low- and intermediate-mass stars (Peterson \& Megeath 2008). It lies in the northern part of the extended Orion Nebula Cluster and is embedded in a 2 pc long, narrow filament extending away from the Orion Nebula itself (Chini et al. 1997; Carpenter 2000; S. T. Megeath et al., in preparation). OMC 2 contains some of the most luminous infrared and submillimeter sources in the Orion A molecular cloud outside of the Orion Nebula (Johnson et al. 1990; Mezger et al. 1990). Over the last few decades, several surveys from infrared to radio wavelengths disentangled the multitudes of sources found in this region, revealing young stellar objects in different evolutionary stages, ranging from deeply embedded protostars to young stars surrounded by disks (Gatley et al. 1974; Rayner et al. 1989; Johnson et al. 1990; Mezger et al. 1990; Jones et al. 1994; Ali \& DePoy 1995; Chini et al. 1997; Lis et al. 1998; Reipurth et al. 1999; Nielbock et al. 2003; Tsujimoto et al. 2003; Peterson \& Megeath 2008; Megeath et al. 2012; Adams et al. 2012).

The first near-IR images of OMC 2 by Gatley et al. (1974) revealed a small cluster of five bright IR sources in a region

\footnotetext{
${ }^{10}$ Visitor at the Infrared Processing and Analysis Center, California Institute of Technology, 770 S. Wilson Ave., Pasadena, CA 91125, USA.

${ }^{11}$ Current address: Department of Physics and Astronomy, Oberlin College, 110 North Professor Street, Oberlin, OH 44074, USA.

12 Hubble Fellow.
}

$90^{\prime \prime}$, or $0.2 \mathrm{pc}$, in diameter. These have subsequently been shown to be young stellar objects with luminosities ranging from 20 to $300 L_{\odot}$ (Adams et al. 2012). Submillimeter and millimeter imaging (Mezger et al. 1990; Chini et al. 1997; Lis et al. 1998) showed that in the center of this small cluster is a bright submillimeter source. This object, OMC-2 FIR 4, is the brightest submillimeter $(350-1300 \mu \mathrm{m})$ source in the OMC 2 region. It is connected through filamentary structures to two other adjacent sources that are bright at submillimeter wavelengths and are coincident with two of the bright IR sources of Gatley et al. (1974): OMC-2 FIR 3 matches a protostar $~ 28^{\prime \prime}$ to the north (also known as SOF $2 \mathrm{~N}$ or HOPS 370 ), while OMC-2 FIR 5 agrees with a protostar $\sim 17^{\prime \prime}$ to the south (SOF 4 or HOPS 369; see Adams et al. 2012). Outside of the massive star-forming region OMC-1 in the Orion Nebula, FIR 4 is the brightest $870 \mu \mathrm{m}$ source in the Orion A cloud (T. Stanke et al. 2014, in preparation). Although bright in the submillimeter, FIR 4 was not detected in the near-IR by Tsujimoto et al. (2003) and only tentatively associated with a near- to mid-IR source by Nielbock et al. (2003). The detection of a $3.6 \mathrm{~cm}$ source with the Very Large Array (VLA) toward FIR 4 was the first compelling evidence that the submillimeter source contained a deeply embedded protostar; the elongated radio source was interpreted as free-free emission originating from shock-ionized gas in an outflow launched by a protostar (Reipurth et al. 1999).

FIR 4 also coincides with the IRAS source 05329-0512. Its bolometric luminosity, integrated over an area of $50^{\prime \prime} \times 50^{\prime \prime}$ 
around it, was estimated to be $420 L_{\odot}$ (Mezger et al. 1990). FIR 4 was thus identified and studied as an intermediate-mass protostar (Johnstone et al. 2003; Crimier et al. 2009). Crimier et al. (2009) constructed a spectral energy distribution (SED) for FIR 4 by retrieving archived mid-infrared to millimeter observations and extracting fluxes. They modeled the SED and derived a total luminosity of $1000 L_{\odot}$. More recently, the infrared emission from a protostar toward FIR 4 (known as SOF 3 or HOPS 108) was resolved by Adams et al. (2012) using $2^{\prime \prime}$ to $19^{\prime \prime}$ resolution data from the Spitzer Space Telescope (Werner et al. 2004), the Stratospheric Observatory For Infrared Astronomy (SOFIA; Young et al. 2012), the Herschel Space Telescope ${ }^{13}$ (Pilbratt et al. 2010), and from the Atacama Pathfinder Experiment (APEX) telescope. This work has cast doubt on the high luminosity of OMC-2 FIR 4; modeling of the SEDs by Adams et al. (2012) found that the intrinsic luminosity lies in the $30-50 L_{\odot}$ range. These data also showed that within the beam of IRAS, the other near-IR sources originally found by Gatley et al. (1974) dominate the flux out to $70 \mu \mathrm{m}$ with luminosities varying from 20 to $300 L_{\odot}$; OMC-2 FIR 3 (SOF $2 \mathrm{~N}$, HOPS 370) was found to be the most luminous source in the region.

Only at wavelengths $\gtrsim 160 \mu \mathrm{m}$ does FIR 4 dominate; however, it is unclear whether the entire submillimeter emission is associated with the protostar observed at shorter wavelengths. Millimeter interferometry by Shimajiri et al. (2008) resolved FIR 4 into 11 dusty cores. Furthermore, they found that highvelocity gas traced by $\mathrm{CO}$ is dominated by an outflow from FIR 3. They proposed that the motion seen in the dense gas toward FIR 4 could be explained by the interaction of the powerful outflow from FIR 3 with the FIR 4 clump. On the basis of interferometric observations made in both continuum and line, López-Sepulcre et al. (2013) interpreted FIR 4 as containing three distinct components, a western core, a southern core and a main core containing a young star, with a total mass of 9.2-25.7 $M_{\odot}$. Noting the lack of detection of outflow signatures from FIR 4 in their interferometric observations and the proposal of Shimajiri et al. (2008) that motions in FIR 4 are driven by an outflow from FIR 3, they suggested that the $3.6 \mathrm{~cm}$ source is due to photo-ionization of gas by an early-type (B3-B4) star with a luminosity of 700-1000 $L_{\odot}$ within one of the three components.

In this publication, we use Spitzer, Herschel, and APEX imaging of FIR 4 from 3.6 to $870 \mu \mathrm{m}$ obtained for the Herschel Orion Protostar Survey (HOPS) to study the protostar associated with FIR 4, with the goal of resolving the large uncertainties in the luminosity of the protostar and its relationship to the submillimeter clump. We use these data to measure the SED of the protostar and constrain its bolometric luminosity and temperature, exploring the effect of the choice of aperture size, or the use of point-spread-function (PSF) fitting photometry, on the final result. By using radiative transfer models, we explore the range of possible luminosities and source properties and show that a wide range of luminosities is possible. We also investigate the relationship between the protostar and submillimeter clump and the possibility that much of the submillimeter luminosity is due to external heating. We favor a model that has a deeply embedded protostar with $L<100 L_{\odot}$ driving an outflow, forming on the side of a massive $\left(\sim 30 M_{\odot}\right)$ clump.

\footnotetext{
13 Herschel is an ESA space observatory with science instruments provided by European-led Principal Investigator consortia and with important participation from NASA.
}

Table 1

Photometry of OMC-2 FIR 4

\begin{tabular}{lccl}
\hline \hline Wavelength & $\begin{array}{c}\text { Flux } \\
(J y)\end{array}$ & Aperture Radius & \multicolumn{1}{c}{ Reference } \\
\hline $\mathbf{8} \mu \mathbf{m}$ & $\mathbf{0 . 0 3}$ & $2^{\prime \prime} .4$ & Megeath et al. (2012) \\
$\mathbf{2 4} \mu \mathbf{m}$ & $\mathbf{1 . 5 1 9}$ & PSF & Megeath et al. (2012) \\
$37.1 \mu \mathrm{m}$ & 8.4 & $4^{\prime \prime} .3$ beam & Adams et al. (2012) \\
$\mathbf{7 0} \mu \mathbf{m}$ & $\mathbf{1 3 2 . 5}$ & $9^{\prime \prime} .6$ & This work \\
$\mathbf{7 0} \mu \mathbf{m}$ & $\mathbf{4 0 . 8 1}$ & PSF & This work \\
$\mathbf{1 0 0} \mu \mathbf{m}$ & $\mathbf{2 8 7 . 7}$ & $9^{\prime \prime} 6$ & This work \\
$\mathbf{1 6 0} \mu \mathbf{m}$ & $\mathbf{6 1 1 . 2}$ & $12^{\prime \prime} .8$ & This work \\
$\mathbf{1 6 0} \mu \mathbf{m}$ & $\mathbf{2 7 0 . 2}$ & PSF & This work \\
$350 \mu \mathrm{m}$ & 67 & $12^{\prime \prime}$ beam & Lis et al. (1998) \\
$\mathbf{3 5 0} \mu \mathbf{m}$ & $\mathbf{5 4 . 7}$ & $7^{\prime \prime} .34$ & This work \\
$\mathbf{3 5 0} \mu \mathbf{m}$ & $\mathbf{4 3 . 2}$ & $7^{\prime \prime} .34$ beam & This work \\
$850 \mu \mathrm{m}$ & 7.5 & $14^{\prime \prime}$ beam & Johnstone \& Bally (1999) \\
$\mathbf{8 7 0} \mu \mathbf{m}$ & $\mathbf{1 2 . 3}$ & $19^{\prime \prime}$ & This work \\
$\mathbf{8 7 0} \mu \mathbf{m}$ & $\mathbf{8 . 3 9}$ & $19^{\prime \prime}$ beam & This work \\
$1.3 \mathrm{~mm}$ & 8.0 & $50^{\prime \prime} \times 50^{\prime \prime}$ & Mezger et al. (1990) \\
$1.3 \mathrm{~mm}$ & 1.252 & $22^{\prime \prime} \times 17^{\prime \prime}$ & Chini et al. (1997) \\
$2.0 \mathrm{~mm}$ & 1.06 & $4^{\prime \prime} .87 \times 22^{\prime \prime} 73$ & López-Sepulcre et al. (2013) \\
$3.6 \mathrm{~cm}$ & $6.4 \times 10^{-4}$ & $\sim 8^{\prime \prime}$ beam & Reipurth et al. (1999) \\
\hline
\end{tabular}

Notes. The flux values we use for our SED models are shown in bold (see text for details). In the column labeled "aperture radius," "PSF" means that the flux was determined via PSF photometry, and a numerical value followed by "beam" indicates that the flux is the peak beam flux of the source as measured in a beam with the specified FWHM.

\section{DATA OVERVIEW}

In this section, we present our data on OMC-2 FIR 4 and explain how we extracted the photometry in the far-IR and submillimeter. OMC-2 FIR 4 was detected by the Spitzer InfraRed Array Camera (IRAC; Fazio et al. 2004) and Multiband Imaging Photometer for Spitzer (MIPS; Rieke et al. 2004) at 8.0 and $24 \mu \mathrm{m}$, respectively (Megeath et al. 2012; see Figure 1). A mid-infrared spectrum $(5-37 \mu \mathrm{m})$ was also taken using the InfraRed Spectrograph (IRS; Houck et al. 2004) on Spitzer. FIR 4 was also detected at $37.1 \mu \mathrm{m}$ using FORCAST (Herter et al. 2012) on SOFIA (source SOF 3 of Adams et al. 2012). As part of HOPS, a Herschel open-time key program (e.g., Fischer et al. 2010; Stanke et al. 2010; Stutz et al. 2013; Manoj et al. 2013; W. J. Fischer et al. 2014, in preparation; B. Ali et al. 2014, in preparation), it was observed with the Photodetector Array Camera and Spectrometer (PACS; Poglitsch et al. 2010) at 70 and $160 \mu \mathrm{m}$ (see Figure 1). In the HOPS catalog, OMC-2 FIR 4 is source HOPS 108. It was also observed with PACS at $100 \mu \mathrm{m}$ by the Gould Belt Survey (e.g., André et al. 2010). In the submillimeter, OMC 2 was mapped at 350 and $870 \mu \mathrm{m}$ with the SABOCA and LABOCA instruments (Siringo et al. 2010, 2009, respectively) on the APEX telescope (see Figure 1). Table 1 displays the fluxes extracted from these data sets, as well as some measurements from the literature. Details on the data reduction and photometry for the PACS data can be found in B. Ali et al. (2014, in preparation), while details on the measurements of the APEX fluxes can be found in Stutz et al. (2013), A. M. Stutz et al. (2014, in preparation), and T. Stanke et al. (2014, in preparation).

In the Spitzer/IRAC images, OMC-2 FIR 4 is faint, but clearly detected, at $8.0 \mu \mathrm{m}$. Some emission can also be seen at $4.5 \mu \mathrm{m}$ and $5.8 \mu \mathrm{m}$, but there is no well-defined point source at these wavelengths. At $5.8 \mu \mathrm{m}$, there seem to be two emission peaks, one that matches the 8 and $24 \mu \mathrm{m}$ position, and one slightly offset, while at $4.5 \mu \mathrm{m}$ there is a strong emission peak only at 


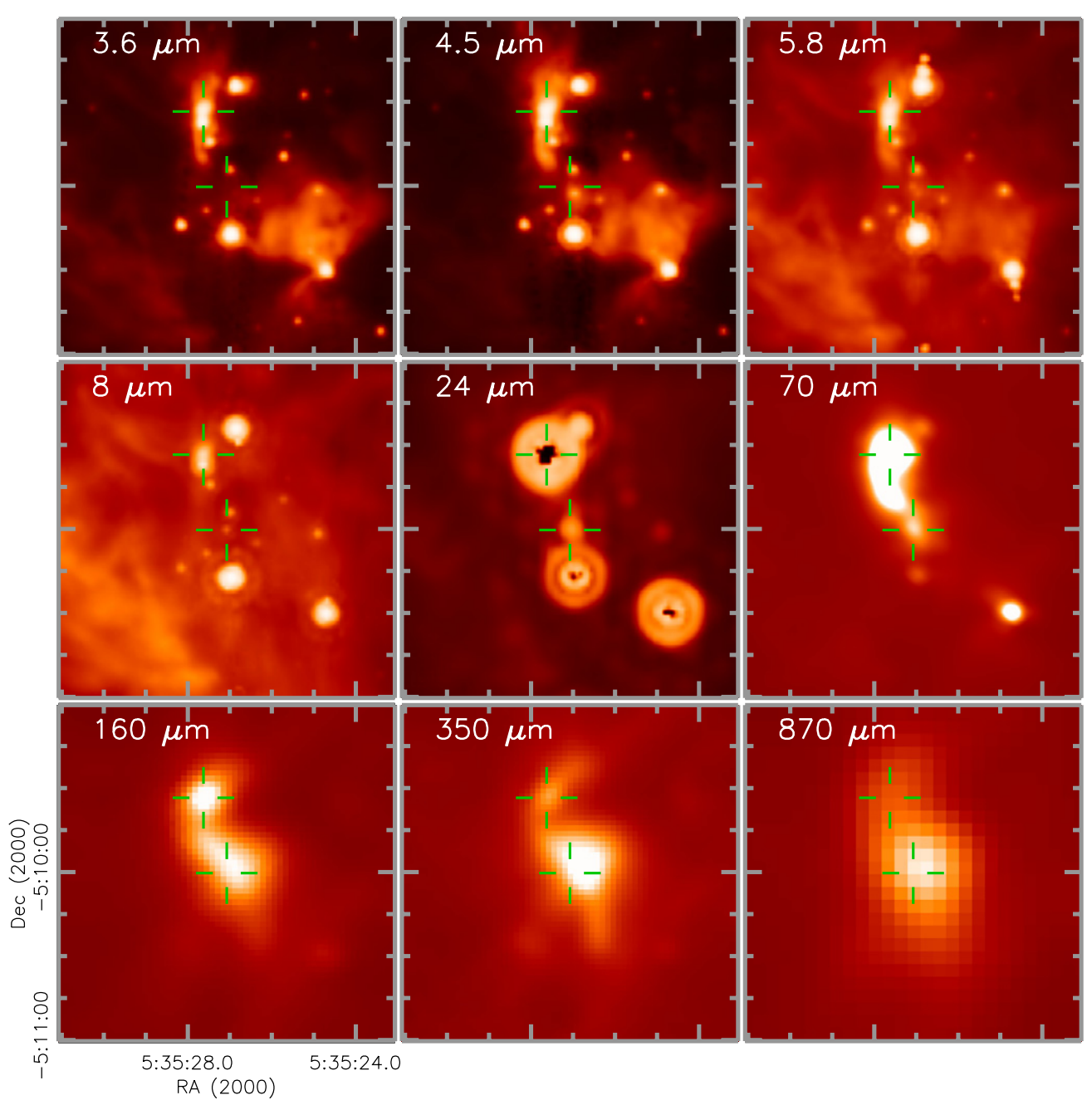

Figure 1. IRAC 3.6, 4.5, 5.8, and $8.0 \mu \mathrm{m}$, MIPS $24 \mu \mathrm{m}$, PACS 70 and $160 \mu \mathrm{m}$, SABOCA $350 \mu \mathrm{m}$, and LABOCA $870 \mu \mathrm{m}$ images of the region around OMC-2 FIR 4 (SOF 3, HOPS 108). At $24 \mu \mathrm{m}$, the saturated sources to the north, south, and southwest are OMC-2 FIR 3 (SOF 2N, HOPS 370), OMC-2 FIR 5 (SOF 4, HOPS 369), and SOF 5 (HOPS 368), respectively. The two crosshairs show the position of FIR 3 (northern crosshairs) and FIR 4 (southern crosshairs).

(A color version of this figure is available in the online journal.)

the offset position. The detection of emission offset relative to the peak position seen at $8-70 \mu \mathrm{m}$ could be an indication of an outflow (see Section 5.4). About 6" to the north of FIR 4 lies an object that is brighter in all IRAC bands, but much fainter at $24 \mu \mathrm{m}$ and not detected at $70 \mu \mathrm{m}$ and longer wavelengths (see Figure 1). This is source MIR 24 tentatively identified with FIR 4 by Nielbock et al. (2003), but it is a separate source (also known as HOPS 64 in the HOPS catalog).

The IRS spectrum of FIR 4 is very noisy in the 5-14 $\mu \mathrm{m}$ region, mostly due to deep ice and silicate absorption features, but at $8 \mu \mathrm{m}$ agrees with the IRAC measurement within $\sim 20 \%$. Given the slit widths of 3".6 for the Short-Low module (SL; 5-14 $\mu \mathrm{m}$ ) and 10'.5 for the Long-Low module (LL; 14-37 $\mu \mathrm{m}$ ), as well as the slit orientations, none of the bright neighboring sources contaminated the IRS spectrum. Only HOPS 64, located 6."3 to the north of FIR 4, partially entered the LL slit, but its flux contribution at wavelengths $\gtrsim 15 \mu \mathrm{m}$ is small (its MIPS $24 \mu \mathrm{m}$ flux is $0.57 \mathrm{Jy}$, compared to $1.5 \mathrm{Jy}$ for FIR 4). There is a discrepancy between the MIPS $24 \mu \mathrm{m}$ flux of FIR 4 and the $24 \mu \mathrm{m}$ flux derived from IRS spectra in that the IRS flux is a factor of two too high. This could be due to the fact that more extended emission from the filament was included in the IRS measurement (slit width of 10 '.5 compared to the typical FWHM of the MIPS $24 \mu \mathrm{m}$ PSF of $\left.\sim 6^{\prime \prime}\right)$. The IRS spectrum was scaled by 0.5 to match the MIPS $24 \mu$ m flux. When compared to the SOFIA/FORCAST measurement at $37.1 \mu \mathrm{m}$ from Adams et al. (2012), where FIR 4 appears as a point source, the IRS spectrum is about a factor of 1.3-1.9 too high (the range considers the calibration uncertainty of the $37.1 \mu \mathrm{m}$ flux), roughly consistent with the discrepancy found for the MIPS $24 \mu \mathrm{m}$ flux.

At 70 and $160 \mu \mathrm{m}$, emission toward OMC-2 FIR 4 can be clearly discerned (Figure 1). FIR 4 is part of a dense filament extending from FIR 3 (HOPS 370) to the north. At $70 \mu \mathrm{m}$, a point source can be seen near the position of the Spitzer 8 and $24 \mu \mathrm{m}$ source. To compare the position of the PACS sources to those in the Spitzer images, the PACS maps have been recentered based on the average offsets between the Spitzer and PACS $70 \mu \mathrm{m}$ observations of the HOPS targets in the field (see Figure 2). The offsets were determined independently for the four distinct images constructed from the four separate groups of PACS scans that covered FIR 4 as part of the HOPS program. In these four images, the $70 \mu \mathrm{m}$ position of FIR 4 is offset from the Spitzer position by 0.'4-1".4 (Figure 2), much smaller than the FWHM of $7^{\prime \prime}$ at $70 \mu \mathrm{m}$. These offsets are comparable to the offsets found for the other HOPS sources in each group and match the positional uncertainty expected from the Herschel 


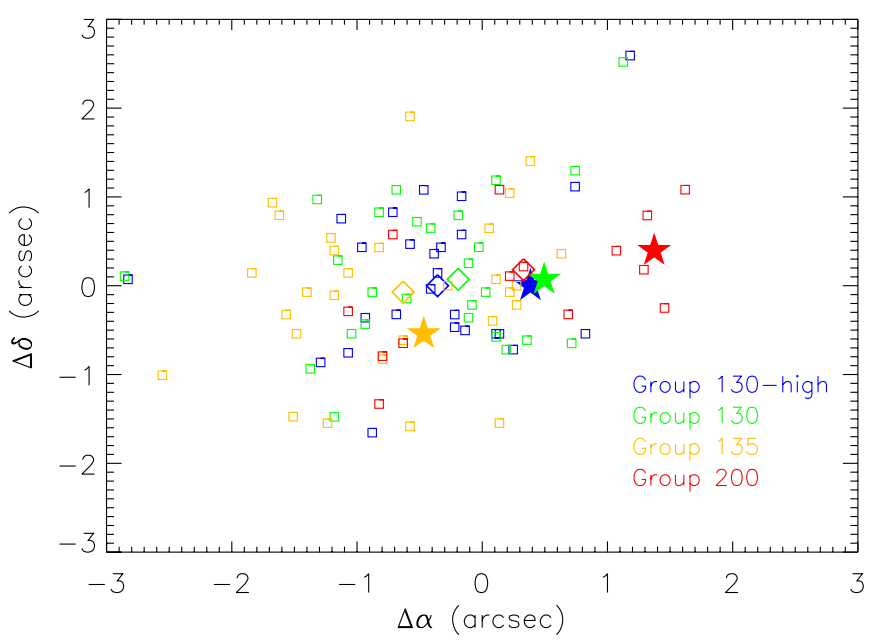

Figure 2. Offsets in right ascension and declination relative to the Spitzer positions for all PACS $70 \mu \mathrm{m}$ sources with Spitzer analogs in the fields that contain the source OMC-2 FIR 4. The targets were observed by Herschel in four different scan groups, represented by four colors. The squares identify all HOPS targets observed in these groups, with FIR 4 shown with a filled star symbol. The open diamonds represent the median offsets found for the objects in the four groups.

(A color version of this figure is available in the online journal.)

pointing accuracy of $\sim 2^{\prime \prime}$. The offset between the Spitzer and PACS $70 \mu \mathrm{m}$ data in right ascension is $\sim 0.7-1^{\prime \prime}$ larger for FIR 4 than the median offset for the other $70 \mu \mathrm{m}$ sources in three of the four images; for the fourth image (constructed from group 135 scans), the offset for FIR 4 and the median offset agree within 0 '.2. However, in each group there are other objects with similar right ascension offsets as FIR 4, so it is not exceptional. Thus, we conclude that the Spitzer 8,24 and $70 \mu \mathrm{m}$ objects are coincident to within the accuracy of our data.

Similarly, the $160 \mu \mathrm{m}$ map was corrected using the offsets derived from the $70 \mu \mathrm{m}$ observations, given that it was observed at the same time. There is an offset between the $70 \mu \mathrm{m}$ source and the peak of the $160 \mu \mathrm{m}$ emission: the brightest part of the extended emission is about $3^{\prime \prime}$ to the northwest of the mid-IR (and $70 \mu \mathrm{m}$ ) position of FIR 4. This $160 \mu \mathrm{m}$ peak also overlaps in position with the peak seen at 350 and $870 \mu \mathrm{m}$, suggesting that, as opposed to the "warm" ( $\leqslant 70 \mu \mathrm{m})$ peak resulting from envelope emission, the "cold" peak could arise from externally heated dust (see Section 5.5). Although there is a clear peak in the $\lambda \geqslant 160 \mu \mathrm{m}$ emission toward FIR4, the source in these bands is markedly extended and is not a point source.

We carried out aperture photometry at 70 and $160 \mu \mathrm{m}$ by centering on the peak of the emission in each band. With aperture photometry at $70 \mu \mathrm{m}$ using an aperture radius of 9.'6, a sky annulus of 9.'6-19.'2, and an aperture correction factor of 1.364 , we derived a flux of $132.5 \mathrm{Jy}$ (the sky emission, i.e., the mode of fluxes inside the sky annulus, amounted to just $0.1 \mathrm{Jy}$ ). However, when applying PSF photometry, ${ }^{14}$ we derived $40.8 \pm$ 4.7 Jy (see B. Ali et al. 2014, in preparation, for details). It

\footnotetext{
14 We used StarFinder for PSF photometry (Diolaiti et al. 2000). StarFinder often finds more than one source within $10^{\prime \prime}$ of the expected source position. FIR 4 was observed four times as part of the HOPS program (four different groups); in two observations StarFinder found multiple sources. In one of these cases, one source was less than $1^{\prime \prime}$ from the expected position, while the other was 8 " away. In the other case StarFinder decomposed the object into three nearby sources. To derive the PSF flux, we only used the data from three observations: we averaged the fluxes from the two observations where only one source was found and from the observation where at least one source was found very close to the expected position.
}

is clear that aperture photometry includes a large contribution of the extended, filamentary emission around FIR 4, so PSF photometry should yield a more reliable source flux at $70 \mu \mathrm{m}$. The residual images from the PSF fits indicated that only a small amount of emission was left around the position of FIR 4. Thus, the flux value from PSF photometry might underestimate the true emission from the protostar at $70 \mu \mathrm{m}$, but not by much.

The PACS $160 \mu \mathrm{m}$ flux is similarly affected by extended emission. FIR 4 is brighter than its northern neighbor FIR 3, but it is embedded in the filament connecting both. Aperture photometry at $160 \mu \mathrm{m}$, centered on the brightness peak at $160 \mu \mathrm{m}$, with an aperture radius of 12 .'. , sky annulus of $12^{\prime \prime} .8-25^{\prime \prime} .6$, and an aperture correction factor of 1.515 , yielded a flux of $611.2 \mathrm{Jy}$ (once again, the sky emission was low, just $1.3 \mathrm{Jy}$ ). On the other hand, PSF photometry ${ }^{15}$ resulted in 270.2 $\pm 8.4 \mathrm{Jy}$. This flux is a better estimate of the envelope emission at $160 \mu \mathrm{m}$, but given that there is no distinct point source at this wavelength, it probably still has a large contribution from extended emission. Also, the positional offset between the 70 and $160 \mu \mathrm{m}$ peak indicated that it is possible that the main contribution to the $160 \mu \mathrm{m}$ emission stems from externally heated dust in a massive core that encompasses the protostar (see Section 5.5), and thus the emission from the envelope itself could be very small. For the PACS $100 \mu \mathrm{m}$ flux, we only had aperture photometry available; adopting the same aperture radius and sky annulus as for the $70 \mu \mathrm{m}$ data, and an aperture correction factor of 1.440 , we derived $287.7 \mathrm{Jy}$ for the flux at $100 \mu \mathrm{m}$. Since this value very likely overestimates the intrinsic flux from FIR 4, we treat it as an upper limit.

The morphology of OMC-2 FIR 4 is similar at 160 and $350 \mu \mathrm{m}$ (Figure 1). It is the brightest object in the area, but embedded in extended emission. To derive a SABOCA $350 \mu \mathrm{m}$ flux for this object, we adopted its beam flux of $43.2 \mathrm{Jy}$ (the SABOCA beam has an FWHM of 7".34), with the centroid determined within a box of size $1.65 \times$ FWHM around the Spitzer position to account for potential offsets. Thus, the beam flux was centered at the brightness peak of the $350 \mu \mathrm{m}$ emission from FIR 4. Aperture photometry with a radius of 3."67 (and no sky subtraction) yielded $29.3 \mathrm{Jy}$; aperture photometry adopting a radius of 7 .'34 and a sky annulus of $11^{\prime \prime} .0-14^{\prime \prime} .7$ resulted in $54.7 \mathrm{Jy}$.

At $870 \mu \mathrm{m}$, FIR 4 is clearly the brightest object, but it is also extended (Figure 1). The LABOCA $870 \mu \mathrm{m}$ beam flux (beam FWHM of 19") amounted to $8.4 \mathrm{Jy}$; also at $870 \mu \mathrm{m}$, the centroid position of the source was determined separately, with the Spitzer position as starting point. Aperture photometry using a radius half the FWHM and no sky subtraction yielded $5.1 \mathrm{Jy}$, while aperture photometry within a radius of $19^{\prime \prime}$ and sky annulus of 28.'5-38" resulted in $12.3 \mathrm{Jy}$. Adams et al. (2012) reported similar SABOCA and LABOCA beam fluxes for FIR 4.

\section{SPECTRAL ENERGY DISTRIBUTION AND BOLOMETRIC LUMINOSITY}

Figure 3 shows the SED of OMC-2 FIR 4 constructed with the data mentioned in the previous section. As discussed earlier, the IRS spectrum displays deep ice absorption features in the 5-8 $\mu \mathrm{m}$ region (due to water ice, methanol, and other organic species), and its silicate absorption feature centered at $10 \mu \mathrm{m}$

\footnotetext{
15 Again, StarFinder found several sources in each of the observations of FIR 4; given the extended nature of FIR 4 at $160 \mu \mathrm{m}$, we adopted the source flux of the brightest object within $8^{\prime \prime}$ of the expected position in each observation and averaged these fluxes.
} 


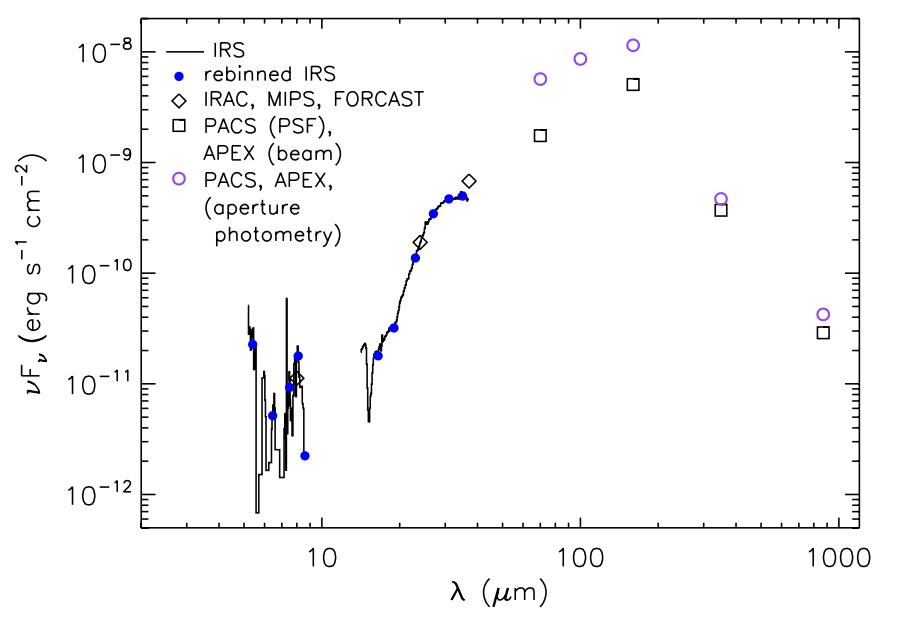

Figure 3. SED of OMC-2 FIR 4 using Spitzer/IRAC, IRS, and MIPS, SOFIA/ FORCAST, Herschel/PACS, and APEX SABOCA and LABOCA data. The small blue dots on the IRS spectrum show the data points that are used in the calculation of $L_{\mathrm{bol}}$ and $T_{\mathrm{bol}}$ and for the model fits. The black diamonds at 8,24 , and $37 \mu \mathrm{m}$ represent the IRAC, MIPS, and FORCAST photometry, respectively. The black squares at 70 and $160 \mu \mathrm{m}$ represent PACS PSF photometry measurements, while the open, purple circles at these wavelengths and at $100 \mu \mathrm{m}$ represent values from aperture photometry. The black squares at 350 and $870 \mu \mathrm{m}$ represent SABOCA and LABOCA beam fluxes, while the open, purple circles at these wavelengths represent aperture photometry values using radii equal to the FWHM of the beams and sky subtraction (see text for details).

(A color version of this figure is available in the online journal.)

is also very deep (with no detected flux over the wavelength interval of maximum absorption). The $\mathrm{CO}_{2}$ ice feature at $15.2 \mu \mathrm{m}$ is, in comparison, less pronounced. The change in slope in the spectrum beyond about $30 \mu \mathrm{m}$ is very likely due to a strong water ice absorption, which is fairly broad and centered at $\sim 45 \mu \mathrm{m}$. The SED plot also shows the fluxes derived from aperture photometry for PACS, SABOCA, and LABOCA data. The difference between aperture and PSF photometry is quite large for PACS fluxes (factors of 3.2 and 2.3 at 70 and $160 \mu \mathrm{m}$, respectively), but smaller at 350 and $870 \mu \mathrm{m}$ (factors of 1.3 and 1.5 , respectively).

As mentioned in Section 2, there seems to be a $\sim 3^{\prime \prime}$ offset in the position of the emission peak between data at $8-70 \mu \mathrm{m}$ and $\gtrsim 160 \mu \mathrm{m}$. When deriving fluxes using aperture photometry, we re-centered at the peak position in each wave band (this is standard procedure for all our targets in the HOPS sample); thus, at longer wavelengths, the aperture was not centered at the same position as was used for the fluxes below $100 \mu \mathrm{m}$. If the far-IR and submillimeter emission is dominated by an externally heated clump of molecular material (which is likely the case; see Section 5.5), then our 100,160, 350, and $870 \mu \mathrm{m}$ fluxes overestimate the emission from the protostar itself and should rather be taken as upper limits. However, using these fluxes gives an idea of the maximum luminosity that could possibly be associated with the protostar OMC-2 FIR 4.

To calculate the bolometric luminosity $\left(L_{\mathrm{bol}}\right)$ from the observed SED, we first rebinned the IRS spectrum to exclude those parts of the spectrum dominated by ices and to smooth over noisy regions (see Figure 3). This resulted in flux values at 5.4, 6.45, $7.5,8.1,8.6,16.5,19.0,23.0,27.0,31.0$, and $35.0 \mu \mathrm{m}$ that trace the continuum emission and part of the short-wavelength wing of the broad silicate absorption feature centered around $10 \mu \mathrm{m}$. If we use the measured Spitzer/IRAC and MIPS photometry, the rebinned version of the IRS spectrum, PSF photometry at 70 and $160 \mu \mathrm{m}$, and the beam fluxes at 350 and $870 \mu \mathrm{m}$, we de-
Table 2

Bolometric Luminosity of OMC-2 FIR 4

\begin{tabular}{lcccccccc}
\hline \hline & \multicolumn{7}{c}{ Data Used for $L_{\text {bol Calculation }}$} \\
\cline { 2 - 8 } \begin{tabular}{l}
$L_{\text {bol }}\left(L_{\odot}\right)$ \\
\cline { 2 - 8 }
\end{tabular} & IRAC & MIPS & IRS & $70 \mu \mathrm{m}$ & $100 \mu \mathrm{m}$ & $160 \mu \mathrm{m}$ & $350 \mu \mathrm{m}$ & $870 \mu \mathrm{m}$ \\
\hline $\mathbf{3 6 . 6}$ & aper & PSF & Yes & PSF & No & PSF & Beam & Beam \\
40.7 & aper & PSF & No & PSF & No & PSF & Beam & Beam \\
78.8 & aper & PSF & Yes & aper & aper & aper & Beam & Beam \\
86.0 & aper & PSF & Yes & aper & aper & aper & aper & aper \\
100.2 & aper & PSF & No & aper & aper & aper & aper & aper \\
\hline
\end{tabular}

Notes. "PSF" means that the flux was determined via PSF photometry, "aper" means that the flux was measured with aperture photometry, including subtraction of a sky value determined in a sky annulus. "no" means that these data were not included in the $L_{\text {bol }}$ calculation. Our preferred $L_{\text {bol }}$ determination is shown in bold.

rive a bolometric luminosity of just $36.6 L_{\odot}$. The corresponding bolometric temperature $\left(T_{\mathrm{bol}}\right)$ is $34.1 \mathrm{~K}$. Neither interpolation of fluxes between the sampled values nor extrapolation of fluxes at wavelengths below $5.4 \mu \mathrm{m}$ and beyond $870 \mu \mathrm{m}$ was done. However, even when we extrapolated the long-wavelength fluxes out to $10 \mathrm{~mm}$ using a power law $F_{v} \propto v^{2}$, we derived the same $L_{\text {bol }}$ value and a $T_{\text {bol }}$ value that is nearly identical, $34.0 \mathrm{~K}$. Since the fluxes in the near-infrared are very small, likely much less than $10 \mathrm{mJy}$, they would not affect the resulting $L_{\text {bol }}$ value.

If we exclude the IRS spectrum from the calculation, we get $40.7 L_{\odot}$ for $L_{\text {bol }}$ and $36.5 \mathrm{~K}$ for $T_{\text {bol }}$. In this case $L_{\text {bol }}$ is slightly higher, since the area under the SED is somewhat larger without the IRS spectrum. If we use the mid-IR photometry, IRS spectrum, submillimeter beam fluxes, but adopt aperture photometry at PACS wavelengths (including the $100 \mu \mathrm{m}$ data point), we calculate $78.8 L_{\odot}$ for $L_{\text {bol }}$ and $36.6 \mathrm{~K}$ for $T_{\text {bol }}$. Finally, adopting aperture photometry at both PACS and APEX wavelengths (with the latter using the aperture radius equal to the FWHM of the beam and sky subtraction), we derive $L_{\text {bol }}=$ $86.0 L_{\odot}$ and $T_{\text {bol }}=33.8 \mathrm{~K}$ (these values change to $100.2 L_{\odot}$ and $36.7 \mathrm{~K}$, respectively, if the IRS spectrum is excluded). Our $L_{\text {bol }}$ calculations are summarized in Table 2.

Thus, depending on which measurements are adopted, we derive bolometric luminosities ranging from $37 L_{\odot}$ to $100 L_{\odot}$ for OMC-2 FIR 4. This large range is simply a result of the complex region around this protostar. However, given that emission from the cold, externally heated clump appears to dominate the fluxes at $\lambda>160 \mu \mathrm{m}$ (see Section 5.5), the $L_{\mathrm{bol}}$ value most closely characterizing the protostar is $37 L_{\odot}$. In contrast, the $T_{\text {bol }}$ value shows little dependence on the chosen photometry, ranging from 34 to $37 \mathrm{~K}$.

\section{FITS OF THE SED WITH STANDARD PROTOSTAR MODELS}

Adams et al. (2012) modeled the SED of OMC-2 FIR 4 using IRAC $8.0 \mu \mathrm{m}$, MIPS $24 \mu \mathrm{m}$, SOFIA/FORCAST $37.1 \mu \mathrm{m}$, PACS 70 and $160 \mu \mathrm{m}$, and APEX 350 and $870 \mu \mathrm{m}$ data. Their reported PACS and APEX fluxes were extracted from an earlier version of the reduced maps. They adopted the sheet collapse model for the envelope from Hartmann et al. (1996) and included the accretion disk model from D'Alessio et al. (1999, 2006). The outflow cavities in the envelope were assumed to follow the streamlines of infalling particles. Depending on whether they included the $160 \mu \mathrm{m}$ data point as an upper limit, they 
Table 3

Models for OMC-2 FIR 4

\begin{tabular}{|c|c|c|c|c|c|c|c|c|c|c|c|}
\hline Model & $\begin{array}{c}L_{\mathrm{tot}} \\
\left(L_{\odot}\right)\end{array}$ & $\begin{array}{l}R_{\text {disk }} \\
(\mathrm{AU})\end{array}$ & $\begin{array}{c}\rho_{1} \\
\left(\mathrm{~g} \mathrm{~cm}^{-3}\right)\end{array}$ & $\begin{array}{c}\text { Parameters } \\
\rho_{1000} \\
\left(\mathrm{~g} \mathrm{~cm}^{-3}\right)\end{array}$ & cavity & $\begin{array}{c}\theta \\
\left({ }^{\circ}\right)\end{array}$ & $\begin{array}{c}i \\
\left({ }^{\circ}\right)\end{array}$ & $\begin{array}{c}A_{V} \\
(\mathrm{mag})\end{array}$ & $R$ & $\begin{array}{l}L_{\text {bol }} \\
\left(L_{\odot}\right)\end{array}$ & $\begin{array}{l}T_{\text {bol }} \\
(\mathrm{K})\end{array}$ \\
\hline Model 1 & 416 & 100 & $7.5 \times 10^{-13}$ & $2.4 \times 10^{-17}$ & Poly & 45 & 70 & 23.9 & 3.81 & 25 & 43 \\
\hline Model 2 & 234 & 500 & $1.9 \times 10^{-12}$ & $5.9 \times 10^{-17}$ & Poly & 45 & 63 & 0.0 & 4.03 & 30 & 41 \\
\hline Model 3 & 54 & 500 & $5.6 \times 10^{-13}$ & $1.8 \times 10^{-17}$ & Poly & 5 & 32 & 0.0 & 6.02 & 58 & 43 \\
\hline Model 4 & 15 & 10 & $1.1 \times 10^{-13}$ & $3.3 \times 10^{-18}$ & Poly & 5 & 49 & 0.0 & 3.03 & 14 & 57 \\
\hline Model 5 & 275 & 500 & $8.3 \times 10^{-13}$ & $2.6 \times 10^{-17}$ & Poly & 35 & 63 & 0.0 & 4.01 & 59 & 42 \\
\hline Model 6 & 23 & 500 & $7.5 \times 10^{-14}$ & $2.4 \times 10^{-18}$ & Poly & 10 & 70 & 0.0 & 3.04 & 14 & 56 \\
\hline Model 7 & 72 & 500 & $7.5 \times 10^{-13}$ & $2.4 \times 10^{-17}$ & Stream & 25 & 32 & 0.0 & 4.32 & 52 & 43 \\
\hline Model 8 & 14 & 10 & $9.8 \times 10^{-14}$ & $3.1 \times 10^{-18}$ & Stream & 5 & 49 & 0.0 & 4.41 & 14 & 59 \\
\hline Model 9 & 540 & 500 & $6.0 \times 10^{-13}$ & $1.9 \times 10^{-17}$ & Stream & 50 & 63 & 0.0 & 8.34 & 53 & 43 \\
\hline Model 10 & 19 & 50 & $7.5 \times 10^{-14}$ & $2.4 \times 10^{-18}$ & Stream & 15 & 87 & 0.0 & 2.85 & 12 & 60 \\
\hline
\end{tabular}

Notes. The model parameters are as follows: $L_{\text {tot }}$ is the total luminosity (which is the sum of the stellar and accretion luminosity), $R_{\text {disk }}$ is the disk radius (which is equal to the centrifugal radius), $\rho_{1}$ and $\rho_{1000}$ are the reference density at 1 and 1000 AU, respectively, $\theta$ is the cavity opening angle, $i$ is the inclination angle, $A_{V}$ is the foreground extinction along the line of sight, and $R$ is a measure for the goodness-of-fit. The column labeled "cavity" describes the cavity shape: "poly" for polynomial, "stream" for streamline. For a polynomial-shaped cavity, the cavity shape exponent is 1.5 . The $L_{\mathrm{bol}}$ and $T_{\mathrm{bol}}$ values were measured by using the fluxes of the individual model SEDs. Note that for all models the stellar radius is $6.61 R_{\odot}$, the stellar luminosity $10.0 L_{\odot}$, the stellar mass $0.5 M_{\odot}$, the disk mass $0.05 M_{\odot}$, the disk scale height exponent 1.25 , and the disk density exponent 2.25 . The outer envelope radius is set to 10,000 AU. Models in italics are those that, at longer wavelengths, fit aperture photometry at 70, 100, 160, 350, and 870 $\mu \mathrm{m}$, while the other models fit the PSF photometry value at $70 \mu \mathrm{m}$ (and, in the case of Models 1 and 2, also PSF photometry at $160 \mu \mathrm{m}$ and the beam fluxes at 350 and $870 \mu \mathrm{m})$.

obtained a total intrinsic luminosity ${ }^{16}$ of $50 L_{\odot}\left(30 L_{\odot}\right.$ with an upper limit at $160 \mu \mathrm{m})$, an inclination angle of $50^{\circ}\left(70^{\circ}\right)$, a cavity opening angle of $8^{\circ}$, an envelope radius of $5000 \mathrm{AU}$, an envelope reference density $\rho_{1}$ (see Kenyon et al. 1993) ${ }^{17}$ of $20(5) \times 10^{-13} \mathrm{~g} \mathrm{~cm}^{-3}$, and an envelope mass of 10 (2.5) $M_{\odot}$.

In E. Furlan et al. (2014, in preparation), we use models from a grid developed for the HOPS program to find the best-fit model of OMC-2 FIR 4 based on an $R$ statistic (Fischer et al. 2012). These models were calculated using the Monte Carlo radiative transfer code developed by Whitney et al. (2003a, 2003b). They use the solution of a rotating, infalling cloud core from Terebey et al. (1984; hereafter TSC model); the disk embedded in the envelope is described by a density power law and a flaring angle exponent. The dust opacities were adopted from Ormel et al. (2011), which include large, icy grains. As opposed to Adams et al. (2012), the cavity carved out in the envelope by the outflows was assumed to have a power law shape (exponent of 1.5; Whitney et al. 2003b), not conical as in the case of a streamline cavity.

For the SED fit in E. Furlan et al. (2014, in preparation), we used the IRAC, MIPS, rebinned IRS fluxes (as described in Section 3), the fluxes from PSF photometry at 70 and $160 \mu \mathrm{m}$, and the beam fluxes at 350 and $870 \mu \mathrm{m}$. The best-fit model from the grid resulted in a total intrinsic luminosity $L_{\text {tot }}$ of $416 L_{\odot}$, an inclination angle of $70^{\circ}$, a cavity opening angle of $45^{\circ}$, and an envelope reference density $\rho_{1}$ of $7.5 \times 10^{-13} \mathrm{~g} \mathrm{~cm}^{-3}$ (see Model 1 in Figure 4(a) and Table 3). This model also required a substantial foreground extinction of $A_{V}=23.9$. However, the next-best model from the grid resulted in no foreground extinction, a somewhat lower inclination angle

\footnotetext{
16 The total luminosity is the intrinsic luminosity derived from the model fits to the SED; it usually differs from the bolometric luminosity, which is derived by integrating the observed SED and thus depends on, e.g., the inclination angle of the source along the line of sight (see, e.g., Whitney et al. 2003b).

17 The reference density $\rho_{1}$ acts as a scaling factor for the envelope density, which determines the thermal emission from the envelope; a $\rho_{1}$ value of $\sim 4 \times 10^{-14} \mathrm{~g} \mathrm{~cm}^{-3}$ roughly divides protostars into Class 0 and I objects (Furlan et al. 2008; Stutz et al. 2013).
}

( $i=63^{\circ}$ ), $L_{\text {tot }}$ of $234 L_{\odot}$, the same cavity opening angle, and a $\rho_{1}$ value of $1.9 \times 10^{-12} \mathrm{~g} \mathrm{~cm}^{-3}$ (see Model 2 in Figure 4 (a) and Table 3). Thus, the total luminosity of the source derived from models strongly depends on the amount of assumed foreground extinction; with an $A_{V}$ value of 23.9 as opposed to 0 , the total luminosity changed by almost a factor of two.

We also ran new model calculations using the same Monte Carlo code of Whitney et al. (2003a, 2003b) for a more indepth exploration of the parameter space. As a starting point for our input files with the model parameters, we used the files from the HOPS model grid; thus, many parameters that we did not adjust, such as the stellar mass and disk density exponent, are the same. We first used a polynomial-shaped cavity, as in our model grid, then a streamline-shaped cavity, since the cavity shape can have a large effect on the resulting model SED (especially if the cavity opening angle is large), but for FIR 4 is not constrained by observations. Similarly, the inclination angle is not constrained, so we explored two sets of models, one with lower inclination angles and one with a more edge-on orientation. We also assumed no foreground extinction; substantial extinction along the line of sight would result in higher total luminosities and change other model parameters, in particular the inclination angle, given that extinction affects mostly the near- and mid-infrared fluxes (see Figure 4(a)).

In panels (b) and (c) of Figure 4, we show the model fits assuming a polynomial-shaped cavity for more face-on and more inclined models, respectively. Each panel shows two model fits each, one that considers the long-wavelength aperture photometry (purple lines), and one that only tries to fit the mid-infrared data and the PSF photometry value at $70 \mu \mathrm{m}$ (cyan lines). As can be seen from Table 3 (Models 3-6), the total luminosity varies widely, depending on which data points are modeled and which orientation along the line of sight is assumed. Models that take aperture photometry at $70 \mu \mathrm{m}$ and beyond into account (Models 3 and 5) have higher $L_{\text {tot }}$ values and higher envelope densities than models that consider only the $70 \mu \mathrm{m}$ PSF photometry value at long wavelengths (Models 4 

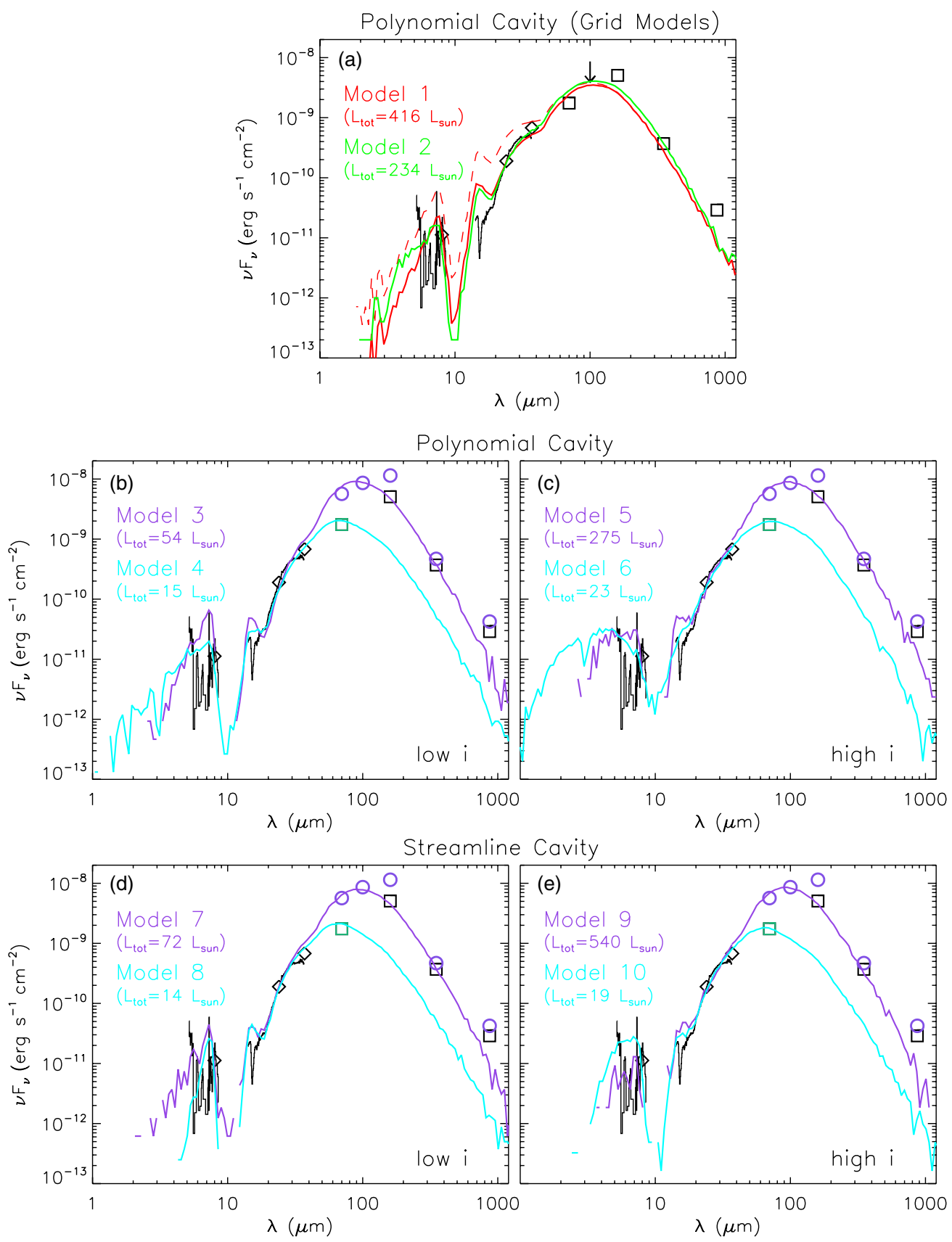

Figure 4. SED of OMC-2 FIR 4 from Figure 3, shown with 10 different model fits (the lines representing the models use fluxes from three apertures: $4^{\prime \prime}$ for $\lambda<8 \mu$ m, $5^{\prime \prime}$ for $\lambda=8-37 \mu \mathrm{m}$, and $10^{\prime \prime}$ for $\lambda>37 \mu \mathrm{m}$ ). (a) Best-fit model from the model grid from E. Furlan et al. (2014, in preparation), which uses polynomial-shaped cavities (Model 1, red solid line; the dashed line shows this same model, but with $A_{V}$ set to 0); best-fit model from the model grid with $A_{V}=0$ (Model 2 , green line). ((b) and (c)) Model fits using polynomial-shaped cavities, one set for low and one set for high inclination angles: fits to the far-IR and submillimeter aperture photometry values, shown with the open circles (Models 3 and 5, purple lines); fits focusing only on the mid-infrared data and the PACS $70 \mu \mathrm{m}$ PSF photometry value, shown as the green square (Models 4 and 6, cyan lines). (d) and (e) Similar to (b) and (c), but using models with streamline-shaped cavities. See text for details and Table 3 for model parameters.

(A color version of this figure is available in the online journal.)

and 6). Also, more edge-on models require total luminosities that are larger than those for models with $i \sim 30^{\circ}-50^{\circ}$. These highinclination models also have larger cavity opening angles. The reference density for models fitting the same data sets changes by about $50 \%$ between the two sets of inclination angles.

A similar result applies to the four models that assume a streamline-shaped cavity (Figures 4(d) and (e), Models 7-10 in Table 3). Compared to the models with polynomial-shaped cavities, the models fitting fluxes from aperture photometry at long wavelengths (Models 7 and 9 versus Models 3 and 5) have larger $L_{\text {tot }}$ values and cavity opening angles, while these parameters are quite similar for the models fitting just the mid-infrared fluxes and the flux from PSF photometry at $70 \mu \mathrm{m}$ (Models 8 and 10 versus Models 4 and 6). Thus, to reproduce large flux values in the far-IR and a steeply rising SED in the mid-IR, a model with a streamline-shaped cavity requires a larger cavity opening angle and a much higher total luminosity than models with polynomial-shaped cavities. 

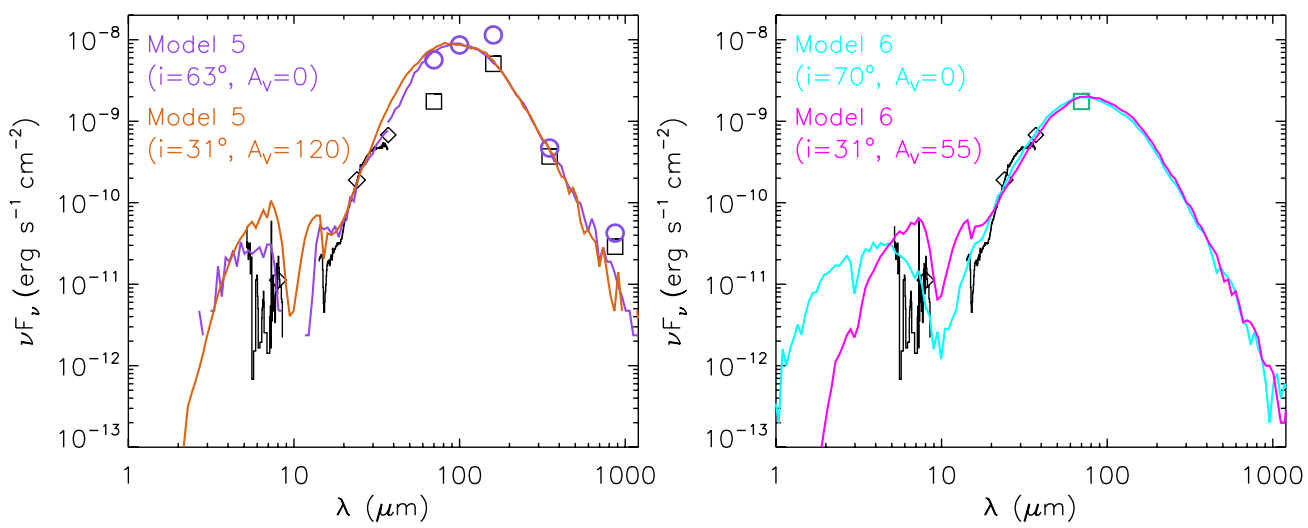

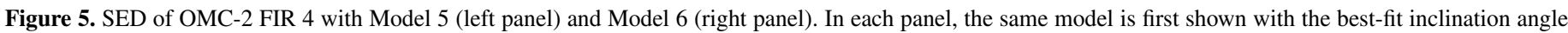
and $A_{V}=0$ (purple and cyan lines), then with a low inclination angle and a relatively high foreground extinction (orange and magenta lines).

(A color version of this figure is available in the online journal.)

This latter type of cavity evacuates more material in the inner envelope than a streamline-shaped cavity, and as a result more shorter-wavelength photons can reach the observer. In the extreme case of Model 9, where $L_{\text {tot }}=540 L_{\odot}$ is required, the streamline-shaped cavity also has to have an opening angle of $50^{\circ}$ such that sufficient infrared flux escapes from the envelope. Interestingly, the models with the streamline-shaped cavity have envelope densities similar to those of the models with the polynomial-shaped cavity. The higher-luminosity models still require envelopes denser by up to an order of magnitude.

For each of the models, Table 3 lists their $R$ value, which is a measure for the goodness-of-fit introduced by Fischer et al. (2012; see also E. Furlan et al. 2014, in preparation). $R$ is defined as follows: $R=(1 / N) \sum_{i=1}^{N} w_{i} \mid \ln \left(F_{\text {obs }}\left(\lambda_{i}\right)-\ln \left(F_{\text {mod }}\left(\lambda_{i}\right) \mid\right.\right.$, where $w_{i}$ is the weight for each data point, $F_{\text {obs }}$ and $F_{\text {mod }}$ are the observed and model fluxes, respectively, and the sum is over the number of data points. The weights were set to the inverse of the approximate fractional uncertainty of each flux measurement and ranged from $1 / 0.04$ to $1 / 0.4$ (with more weight given to the 3-70 $\mu \mathrm{m}$ region; see E. Furlan et al. 2014, in preparation, for details). When calculating $R$ values for the various models, we used the same photometry values and rebinned IRS fluxes (see Figure 3 and Section 3) in the mid-IR, but at longer wavelengths we included aperture photometry at $\lambda \geqslant 70 \mu \mathrm{m}$ for Models 3, 5,7 , and 9 , and only the $70 \mu \mathrm{m}$ PSF photometry at $70 \mu \mathrm{m}$ for Models 4, 6, 8, and 10. Thus, the $R$ values for the latter set of models are in general lower than those for the former set. Overall, most models have $R$ values in the 3-4 range; from a visual inspection of Figure 4, they are indeed quite comparable, with the most noticeable differences in the near-IR and in the depth of the $10 \mu \mathrm{m}$ silicate feature, where there is little to no emission in both the observed and modeled fluxes (and thus they do not have a measurable effect on the $R$ value).

So far, the models calculated for this work do not include any foreground extinction. As mentioned earlier and shown in Figure 4(a), extinction will depress the near- and mid-IR fluxes and leave the far-IR and submillimeter fluxes unchanged. Thus, its effect is similar to that of the inclination angle; dust in a highly inclined envelope will cause more extinction of photons on their way to the observer. In Figure 5 we explored the effect of foreground extinction on two of the high-inclination models (Models 5 and 6). They are shown with their best-fit inclination angle $\left(63^{\circ}\right.$ and $70^{\circ}$, respectively) and no additional extinction along the line of sight, and with a low inclination angle of $31^{\circ}$ and foreground extinction large enough such that the model fluxes still roughly reproduce the observed SED. With a low inclination angle, Model 5 requires $A_{V}=120$, while Model 6 needs $A_{V}=55$. Even though these latter models could benefit from tweaking some parameters, they demonstrate that inclination angle and foreground extinction are highly degenerate parameters. Thus, our models with high luminosity either require a large foreground extinction or a more edge-on orientation.

To examine the effect of a significant contribution from extended, externally heated emission to the far-infrared and submillimeter fluxes, we used a model that fits the mid-infrared data points and the PSF photometry at $70 \mu \mathrm{m}$ and added a modified blackbody (for the latter, we used the same dust opacities from Ormel et al. (2011) adopted for our models). All the other models presented so far do not include such a component; the long-wavelength fluxes are just fit by emission from the disk and envelope. We chose Model 4 to represent the protostar, but since Models 4, 6, 8, and 10 all have similar SEDs in the $20-1000 \mu \mathrm{m}$ range and $L_{\text {bol }}$ values of $12-14 L_{\odot}$, the choice of model does not affect the results. With the combined protostar and blackbody model, we aimed to fit the aperture photometry fluxes at long wavelengths, since they are most likely dominated by extended emission. The best-fit combination of Model 4 and a modified blackbody is shown in Figure 6; it requires a blackbody temperature of $18.5 \mathrm{~K}$. The PACS 70, 100, and $160 \mu \mathrm{m}$ fluxes are fit well, while the $350 \mu \mathrm{m}$ flux is overestimated and the $870 \mu \mathrm{m}$ flux slightly underestimated. The discrepancy at $350 \mu \mathrm{m}$ is likely an aperture effect, given the small aperture used at this wavelength. The combined bolometric luminosity of Model 4 and the $18.5 \mathrm{~K}$ blackbody amounts to $76 L_{\odot}$, which is very similar to the $L_{\text {bol }}$ value measured from the observed SED with the mid-IR data, aperture photometry at 70,100 , and $160 \mu \mathrm{m}$, and the beam fluxes in the submillimeter (see Section 3). The contribution of the modified blackbody to this $L_{\text {bol }}$ value is $62 L_{\odot}$, leaving just $14 L_{\odot}$ as $L_{\text {bol }}$ for the protostar.

\section{DISCUSSION}

\subsection{The Bolometric Luminosity of the Protostar OMC-2 FIR 4}

Our new measurements of OMC-2 FIR 4 in the far-IR and submillimeter suggest that its bolometric luminosity is far below the most recent value of $\sim 1000 L_{\odot}$ suggested in the literature: we derive a range from $37 L_{\odot}$ to $\sim 100 L_{\odot}$, with the former value more likely to describe the protostar, since it is derived 


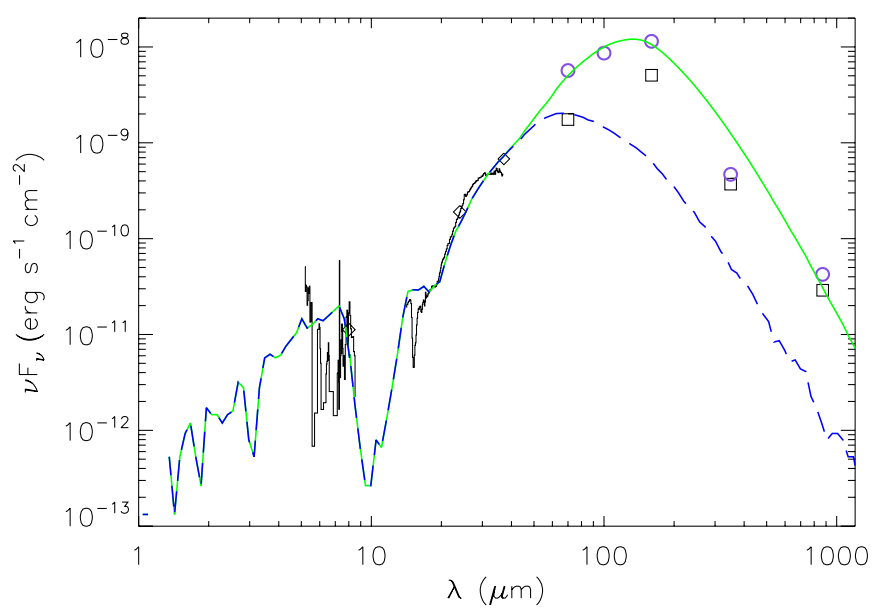

Figure 6. SED of OMC-2 FIR 4 as in Figures 4(b)-(e) shown with a model composed of Model 4 (blue dashed line; see also Figure 4(b)) and a modified blackbody with a temperature of $18.5 \mathrm{~K}$; the combined fluxes are shown with the green solid line.

(A color version of this figure is available in the online journal.)

using smaller apertures for the source fluxes. Also, given that the IRS spectrum plays an important role in constraining the midinfrared part of the SED, the more realistic upper limit for $L_{\mathrm{bol}}$ is $86 L_{\odot}$ (which is derived when including the IRS spectrum in the SED).

As noted by López-Sepulcre et al. (2013), the fluxes and thus luminosity of OMC-2 FIR 4 depend strongly on which apertures are used. The larger the aperture, the more envelope emission, but also extended emission from the surrounding filament and emission from neighboring sources is included. We find that, for isolated protostars in the Orion star-forming region (distance of 420 pc; Menten et al. 2007; Hirota et al. 2007), aperture radii of $\sim 10^{\prime \prime}$ in the far-IR $(70-160 \mu \mathrm{m})$ capture most of the emission from the envelope at these wavelengths (see E. Furlan et al. 2014, in preparation). Choosing larger radii risks including surrounding emission that is not associated with the envelope. Crimier et al. (2009) likely overestimated the fluxes of FIR 4, since they integrated their derived continuum profiles at 350 , 450 , and $850 \mu \mathrm{m}$ out to $\sim 20^{\prime \prime}$, their derived envelope size. However, FIR 4 seems to be surrounded by extended emission, and a $20^{\prime \prime}$ aperture will include emission from this surrounding material. Their derived fluxes at 350 and $850 \mu \mathrm{m}$ are about an order of magnitude larger than our APEX fluxes at similar wavelengths. Crimier et al. (2009) also extracted IRAS fluxes at 60 and $100 \mu \mathrm{m}$; however, the IRAS beam is very large at these wavelengths, and contamination by FIR 3 and FIR 5 likely also plays a role. Their extracted MIPS $24 \mu \mathrm{m}$ flux is also overestimated due to the aperture radius of $15^{\prime \prime}$; their flux value of $5.0 \mathrm{Jy}$ is 3.3 times larger than the value derived by Megeath et al. (2012) with PSF fitting.

The big discrepancy in flux measurements resulting from adopting apertures that are at most a factor of a few different suggests that the region around FIR 4 is very complex and contains copious amounts of extended emission. The dust in this extended material is likely to be heated by the strong far-IR radiation field present in the Orion cloud complex, and is not internally heated by the protostar itself. Thus, if we want to characterize the protostar itself, it seems reasonable to adopt conservative (i.e., smaller) aperture sizes to measure fluxes. The offset we found for the emission peak at $\lambda \leqslant 70 \mu \mathrm{m}$ and $\lambda \geqslant 160 \mu \mathrm{m}$ suggests that even our lowest flux values in the $160-870 \mu \mathrm{m}$ region could overestimate the envelope emission. This is supported by the interferometer results of López-Sepulcre et al. (2013), who found that there are multiple components in OMC-2 FIR 4, only one of which contains a protostar. We therefore ignore the more extended core resolved in the observations of Shimajiri et al. (2008) and López-Sepulcre et al. (2013) and focus on the properties of the protostar and its inner ( $\lesssim 8000$ AU radius) envelope. As shown in Section 4, protostellar models that assume a dense, infalling envelope around a single protostar can describe the observed SED. We will discuss the relationship of this protostar to the more extended core in the FIR 4 region in Section 5.5.

\subsection{The Classification of the Protostar OMC-2 FIR 4}

Adopting an $L_{\text {bol }}$ value of $37 L_{\odot}$ for FIR 4 , and using the beam fluxes at 350 and $870 \mu \mathrm{m}$, we can calculate the ratio of submillimeter luminosity $\left(L_{\text {submm }}\right)$ and $L_{\text {bol }}$. For $L_{\text {submm }}$, we integrated the SED at wavelengths $\geqslant 350 \mu \mathrm{m}$ (André et al. 1993). We derived $L_{\text {submm }} / L_{\text {bol }}$ of $2 \%$ (the result is the same if we add a long-wavelength extrapolation to the SED reaching to $10 \mathrm{~mm}$ ). This value is four times larger than the minimum value for a Class 0 protostar (André et al. 1993), so OMC-2 FIR 4 appears to be in a very early evolutionary state, when presumably most of the stellar mass is still in the envelope or core surrounding the protostar. Thus, it shares some of the properties of the PACS Bright Red sources (PBRs) discovered by Stutz et al. (2013), which are among the youngest protostars, with high envelope densities and infall rates. The log of the ratio of its 70 and $24 \mu \mathrm{m}$ flux in $\lambda F_{\lambda}$ space amounts to 0.96 , while for PBRs this ratio is larger than 1.65. Nonetheless, our model fits (Section 4) yielded high envelope densities, $\rho_{1} \geqslant 7.5 \times 10^{-14} \mathrm{~g} \mathrm{~cm}^{-3}$, very similar to those of the PBRs studied in Stutz et al. (2013).

Even model fits that included the $70 \mu \mathrm{m}$ PSF photometry point and no data at longer wavelengths (Models 4, 6, 8, and 10) resulted in $\rho_{1}$ values close to $1.0 \times 10^{-13} \mathrm{~g} \mathrm{~cm}^{-3}$, suggesting that even if we assume that the far-IR and submillimeter emission is dominated by externally heated dust, the derived envelope density of FIR 4 is still large. These models also yielded $L_{\text {bol }}$ values of $12-14 L_{\odot}$ for the protostar, which is less than half the smallest value measured from the observed SED, but is a result of these model fluxes being lower by almost a factor of 10 compared to the beam fluxes at 350 and $870 \mu \mathrm{m}$ and the flux from PSF photometry at $160 \mu \mathrm{m}$ (see Figure 4). The ratios of submillimeter to bolometric luminosity for these models are $0.5 \%-0.6 \%$, on the low end for a Class 0 object (André et al. 1993), but the $T_{\text {bol }}$ values are $\leqslant 60 \mathrm{~K}$ and thus clearly in the Class 0 range (Chen et al. 1995). Therefore, there is strong observational evidence that OMC-2 FIR 4 is a Class 0 protostar. The high envelope density suggests that it is in an early evolutionary stage, and its SED classification as a Class 0 object translates into a Stage 0 physical state (see Robitaille et al. 2006).

\subsection{Determining Source Properties from Models}

As shown in Section 4, a wide range of models can fit the observed fluxes of OMC-2 FIR 4, especially due to the somewhat uncertain source fluxes in the far-infrared and submillimeter and unconstrained model parameters such as the inclination angle and cavity shape. On the other hand, the current model fits can already give rough estimates for some source properties: the envelope density is relatively high, in the $\rho_{1} \sim 10^{-13}-10^{-12} \mathrm{~g} \mathrm{~cm}^{-3}$ (or $\rho_{1000} \sim 3 \times 10^{-18}-3 \times 10^{-17} \mathrm{~g} \mathrm{~cm}^{-3}$ ) range, and the cavity is either relatively narrow, combined with a more face-on 


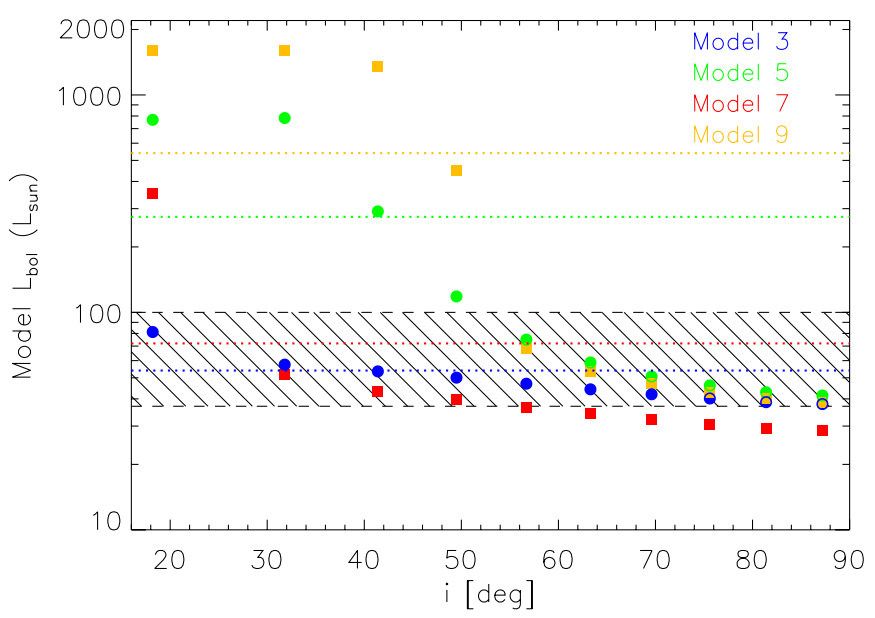

Figure 7. Bolometric luminosity vs. the inclination angle for Models 3 (blue; $\left.L_{\text {tot }}=54 L_{\odot}\right), 5\left(\right.$ green; $\left.L_{\text {tot }}=275 L_{\odot}\right), 7\left(\right.$ red; $\left.L_{\text {tot }}=72 L_{\odot}\right)$, and 9 (yellow; $\left.L_{\text {tot }}=540 L_{\odot}\right)$. The horizontal, dotted lines show the value of $L_{\text {tot }}$ for these four models. The dashed region shows the range of $L_{\mathrm{bol}}$ values derived from the observed SED of OMC-2 FIR 4 (see text for details).

(A color version of this figure is available in the online journal.)

orientation, or wide, if the inclination angle is high or a large amount of foreground extinction is present.

When comparing our Models 7 and 9 to the modeling results of Adams et al. (2012), the envelope reference densities are similar, but our total luminosities and cavity opening angles are larger. Even though all these models assume streamline-shaped cavities, Adams et al. (2012) used the sheet collapse solution for the envelope structure. This results in a wider region of decreased density along the rotation axis (which is the same as the outflow axis) compared to our TSC models, roughly corresponding to a wider cavity. Thus, a sheet-collapse model with a smaller cavity still allows the escape of a large amount of mid-IR photons, even at higher inclination angles. Our models require both higher total luminosities (by up to a factor of $\sim 20$ ) and larger cavity opening angles (by up to a factor of six) to allow sufficient photons to reach the observer.

Inferring the total luminosity for FIR 4 is more difficult. While the bolometric luminosity is derived from the observed SED (assuming isotropic emission), the total luminosity is the intrinsic energy output from the object. Depending on the inclination angle, cavity opening angle, or the amount of foreground extinction, an object with the same $L_{\text {tot }}$ value will have $L_{\text {bol }}$ values that are higher or lower (see Whitney et al. 2003b). Observed fluxes determine $L_{\text {bol }}$, so it is not straightforward to convert it to an $L_{\text {tot }}$ value. With $A_{V}=23.9$, $L_{\text {tot }}$ of FIR 4 can be as high as $416 L_{\odot}$, but also a model with a streamline-shaped cavity, $A_{V}=0$, and a more edgeon orientation can yield $L_{\text {tot }}=540 L_{\odot}$. On the other hand, the $L_{\text {bol }}$ value derived from these two model SEDs is $25 L_{\odot}$ for the former model and $53 L_{\odot}$ for the latter one (see Table 3 ).

In Figure 7, we show the effect of inclination angle on $L_{\text {bol }}$ derived for the fluxes of those models that aim at fitting the aperture photometry values at longer wavelengths (Models 3, 5, 7, and 9). Each model has a certain total luminosity, $L_{\text {tot }}$ (see Table 3), which does not depend on the inclination angle. However, $L_{\mathrm{bol}}$, which is derived from the SED, strongly depends on the viewing angle (see Whitney et al. 2003b, Section 3.3). For more face-on orientations, $L_{\text {bol }}$ is higher than $L_{\text {tot }}$, especially for the higher-luminosity models. At $i \gtrsim 45^{\circ}$, the bolometric luminosity is lower than $L_{\text {tot }}$. Thus, to fit the observed $L_{\text {bol }}$

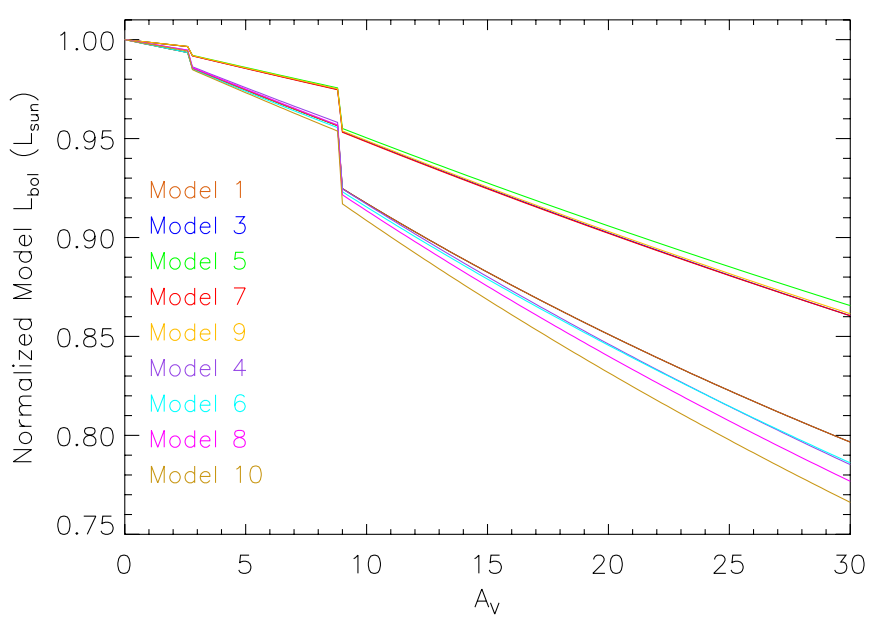

Figure 8. Bolometric luminosity, derived from fluxes attenuated by foreground extinction and normalized to the $L_{\text {bol }}$ value at $A_{V}=0$, vs. the foreground extinction $A_{V}$ for Models 1 (orange), 3 (blue), 5 (green), 7 (red), and 9 (yellow), 4 (purple), 6 (cyan), 8 (magenta), and 10 (beige). The discontinuities at $A_{V}$ values of 2.7 and 9 are due to the transitions to different extinction laws $\left(R_{V}=\right.$ 5 curve from Mathis 1990 for $A_{V}=0-2.7$, and two curves from McClure 2009 for $A_{V}=2.7-9$ and 9-30).

(A color version of this figure is available in the online journal.)

values, a high-luminosity model requires a larger inclination angle than a model with a lower total luminosity. This is reflected in the results presented in Table 3, where the models with the highest luminosity have inclination angles $\geqslant 63^{\circ}$. Given that $L_{\text {bol }}$ of FIR 4 is likely $\sim 40 L_{\odot}$ based on the observations (see Section 3 ), a total luminosity of a few hundred $L_{\odot}$ is only possible if the inclination angle of the object is relatively high.

The effect of foreground extinction on $L_{\text {bol }}$ is less dramatic than that of the inclination angle, as long as $A_{V} \lesssim 30$ (see Figure 8). Using all the models from Table 3 except for Model 2, we calculated $L_{\text {bol }}$ for $A_{V}=0-30$ (i.e., we extinguished the fluxes using $A_{V}$ values ranging from 0 to 30 , then computed $L_{\mathrm{bol}}$ ) and plotted $L_{\text {bol }}$ normalized by its value at $A_{V}=0$. Models 3, 5,7 , and 9 (which fit the higher far-IR and submillimeter flux values) show a nearly identical decline in $L_{\text {bol }}$ with increasing extinction; at $A_{V}=30, L_{\text {bol }}$ is $86 \%$ of its value at $A_{V}=0$. The $L_{\text {bol }}$ values for Models 4, 6, 8, and 10 (which fit the $70 \mu \mathrm{m}$ PSF photometry, but no additional data at longer wavelengths) decrease more steeply, reaching $76 \%-80 \%$ of their extinctionfree values at $A_{V}=30$. The best-fit model from the grid (Model 1) is the only model that included foreground extinction in order to fit the SED; with $A_{V}=0$, its $L_{\text {bol }}$ value is $29 L_{\odot}$, and for the best-fit $A_{V}$ of $23.9, L_{\text {bol }}$ decreases to $25 L_{\odot}$. As mentioned in Section 4, extinction and inclination angle are degenerate parameters; some of our models with $A_{V}=0$ and a large inclination angle could actually be modified to models with lower inclination angles and larger $A_{V}$ values, with hardly any change in $L_{\text {bol }}$, given that $L_{\text {bol }}$ increases for lower inclination angles, but decreases with $A_{V}$.

The total luminosity also depends on which data sets are fit. While the near- and mid-infrared fluxes of the models in Figures 4(b)-(e) are comparable, they are strikingly different in the far-infrared and submillimeter. If we adopt the fluxes from aperture photometry to represent the emission from the envelope at long wavelengths, the envelope is 5-11 times denser than if we use the flux from PSF photometry at $70 \mu \mathrm{m}$. The difference in internal luminosity when fitting these two data sets is a factor of 4-5 for the low-inclination models, but increases to a factor of 


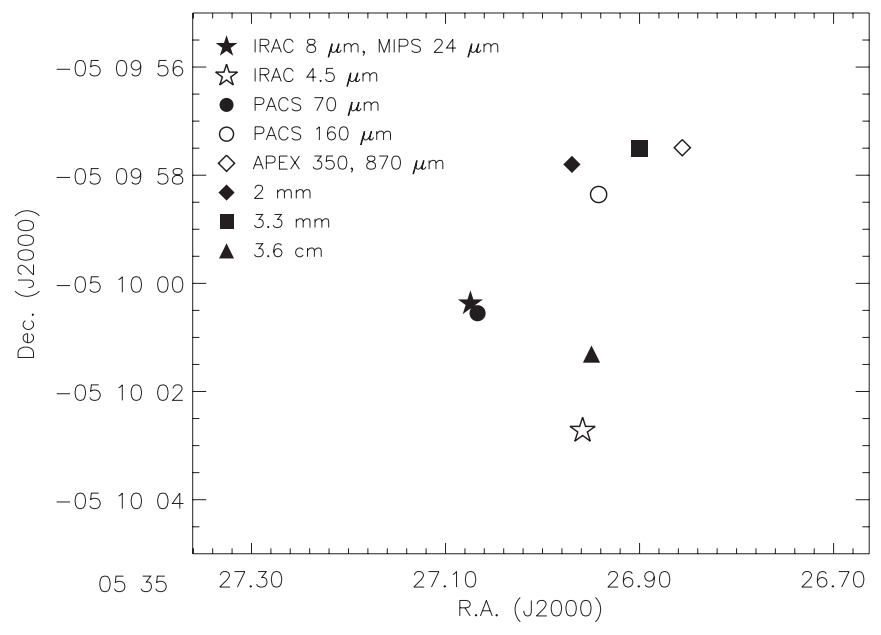

Figure 9. Positions of OMC-2 FIR 4 in the sky in different wave bands. At $4.5 \mu \mathrm{m}$, the position shown is that of the offset peak seen in Figure 1. The positions at 70 and $160 \mu \mathrm{m}$ are those of FIR 4 from scan group 130, corrected by the average offset of HOPS sources in that group relative to their Spitzer positions. The $2 \mathrm{~mm}, 3.3 \mathrm{~mm}$, and $3.6 \mathrm{~cm}$ positions are taken from LópezSepulcre et al. (2013), Shimajiri et al. (2008), and Reipurth et al. (1999), respectively. The coordinate values are listed in Table 4.

12-28 for the high-inclination models. When PSF photometry at $70 \mu \mathrm{m}$ is used, the model fluxes beyond $100 \mu \mathrm{m}$ seem to be too low by about an order of magnitude. The discrepancy between model and data at 160, 350, and $870 \mu \mathrm{m}$ could be explained if the PSF photometry at $160 \mu \mathrm{m}$ and the beam fluxes in the submillimeter were contaminated by extended emission or if the PSF photometry at $70 \mu \mathrm{m}$ underestimated the true envelope emission.

We showed that a protostellar model that only considers fluxes out to $70 \mu \mathrm{m}$ (using the PSF photometry value at that wavelength) and adds a contribution of $\sim 20 \mathrm{~K}$ dust can reproduce the SED that uses aperture photometry fluxes at long wavelengths. In this case, most of the far-IR and submillimeter emission is generated by this extended dust component that is not necessarily part of the envelope when modeling FIR 4. Given that its bolometric luminosity is $62 L_{\odot}$, compared to $14 L_{\odot}$ for the protostar, the dust must be heated by external sources (see also Section 5.1). Thus, if this interpretation of the SED is correct, the protostar associated with OMC-2 FIR 4 is of just moderate luminosity.

\subsection{Does OMC-2 FIR 4 have an Outflow?}

A key piece of evidence to support the interpretation of OMC-2 FIR 4 as a young protostar would be the detection of an outflow. While OMC-2 FIR 3 drives an outflow that likely reaches FIR 4, it is not clear whether FIR 4 itself powers one.

VLA imaging by Reipurth et al. (1999) detected an elongated radio continuum source toward OMC-2 FIR 4 at $3.6 \mathrm{~cm}$; it is the weakest of the three centimeter sources detected in its vicinity, the others are FIR 3 (SOF 2N, HOPS 370) and SOF 5 (also HOPS 368; Adams et al. 2012). They interpreted the centimeter source as free-free emission from shocks in an outflow driven by FIR 4; this is the favored interpretation for centimeter radio jets found toward low mass protostars (Anglada 1996). The location of the radio continuum source encompasses the strong emission peak at $4.5 \mu \mathrm{m}$ and a weaker one at $5.8 \mu \mathrm{m}$, which appear in our data offset relative to the peak position seen at $8-70 \mu \mathrm{m}$ (see Figure 9 and Table 4). Outflow knots can be detected at $4.5 \mu \mathrm{m}$
Table 4

Positions of the Peak Emission from OMC-2 FIR 4 at Different Wavelengths

\begin{tabular}{lccl}
\hline \hline Wavelength & R.A. (J2000) & Decl. (J2000) & \multicolumn{1}{c}{ Reference } \\
\hline $4.5 \mu \mathrm{m}$ & 53526.96 & -51002.7 & Megeath et al. (2012) \\
$8.0,24 \mu \mathrm{m}$ & 53527.07 & -51000.4 & Megeath et al. (2012) \\
$70 \mu \mathrm{m}$ & 53527.07 & -51000.6 & This work \\
$160 \mu \mathrm{m}$ & 53526.94 & -50958.4 & This work \\
$350,870 \mu \mathrm{m}$ & 53526.85 & -50957.5 & This work \\
$2.0 \mathrm{~mm}$ & 53526.97 & -50957.8 & López-Sepulcre et al. (2013) \\
$3.3 \mathrm{~mm}$ & 53526.90 & -50957.5 & Shimajiri et al. (2008) \\
$3.6 \mathrm{~cm}$ & 53526.95 & -51001.3 & Reipurth et al. (1999) \\
\hline
\end{tabular}

Notes. These positions are shown in Figure 9; see the figure caption for notes on the $4.5,70$, and $160 \mu \mathrm{m}$ positions.

and $5.8 \mu \mathrm{m}$ due to shocked $\mathrm{H}_{2}$ emission (Smith \& Rosen 2005), so it is possible that the southwestern lobe of an outflow from FIR 4 has been detected also in the mid-IR. Alternatively, the 4.5 and $5.8 \mu \mathrm{m}$ emission could be light scattered in an outflow cavity (Whitney et al. 2003b).

Anglada $(1995,1996)$ showed that the centimeter flux from low- to intermediate-mass young stars is dominated by collisional ionization in outflow-driven shocks. They found that the centimeter luminosity is correlated with the momentum rate in the outflow $(\dot{P})$, which is in turn correlated with $L_{\text {bol }}$. The resulting empirical relationship between the centimeter luminosity and $L_{\text {bol }}$ for low- to intermediate-mass stars is $S_{\nu} d^{2} /\left(\mathrm{mJy} \mathrm{kpc}^{2}\right)=10^{-2.1}\left(L_{\mathrm{bol}} / L_{\odot}\right)^{0.6}$, where $S_{v}$ is the $\mathrm{cm}$ flux in mJy and $d$ is the distance to the source in $\mathrm{kpc}$ (G. Anglada 2014, private communication). Using the flux densities in Reipurth et al. (1999) and adopting a distance of $420 \mathrm{pc}$, we find $L_{\text {bol }}$ values of $\sim 800,80$, and $190 L_{\odot}$ for FIR 3, FIR 4, and SOF 5, respectively. Thus, the radio continuum flux is most consistent with a $\sim 100 L_{\odot}$ luminosity for FIR 4 , although there is considerable scatter and uncertainty in the relationship relating luminosity to centimeter flux. Just based on its $S_{\nu} d^{2}$ value of 0.11 , the centimeter emission from FIR 4 is consistent with either a jet from a $\sim 100 L_{\odot}$ source (shock ionization) or an $\mathrm{H}$ II region from a $\gtrsim 10^{3} L_{\odot}$ source (photoionization; see Figure 5 of Anglada 1995). Our data and models are in favor of the lower luminosity for FIR 4, thus supporting the interpretation of the centimeter emission as originating in an outflow. Given that $\dot{P} \propto\left(S_{v} d^{2}\right)^{1.1}$ (Anglada 1995), we can also estimate that the outflow of FIR 4 has four times less momentum flux than the outflow driven by FIR 3 (0.64 mJy for FIR 4 versus $2.48 \mathrm{mJy}$ for FIR 3 at $3.6 \mathrm{~cm}$ ).

Alternatively, López-Sepulcre et al. (2013) interpreted the centimeter emission as arising from photoionization from an embedded B3-B4 zero-age main sequence star, which would have a luminosity of 600-1000 $L_{\odot}$. We do not favor this interpretation since there is no supporting evidence for photoionization; the IRS spectrum of the source does not detect the polycyclic aromatic hydrocarbon features that are common in reflection nebulae around intermediate-luminosity stars. However, given the high extinction toward the source and the broad range of plausible luminosities for FIR 4, it is not currently possible to rule out this interpretation.

Additional evidence for an outflow comes from the far-IR CO spectra. Recently, Herschel spectroscopy with PACS has shown that FIR 4 has the highest far-IR CO luminosity in the sample of Orion protostars studied by Manoj et al. (2013). The high-excitation, far-IR molecular line emission appears compact ( $2000 \mathrm{AU})$ and centered on FIR 4. The excitation 
energies and critical densities of the transitions suggest that the far-IR CO emission originates in hot $(T>300 \mathrm{~K})$ gas, with fits to the far-IR CO rotational excitation diagrams yielding temperatures exceeding $2000 \mathrm{~K}$ and relatively low densities of $n\left(\mathrm{H}_{2}\right)<10^{6} \mathrm{~cm}^{-3}$ (Manoj et al. 2013). This hot gas is likely heated by shocks, possibly inside the outflow cavity or along cavity walls. Kama et al. (2013) detected broad wings in the far-infrared lines of $\mathrm{OH}, \mathrm{H}_{2} \mathrm{O}$, and $\mathrm{CO}$ observed with Herschel/HIFI. The line wings were symmetric and their width and strength increased with the excitation level of the line, suggesting emitting gas that is hot or dense and thus possibly a compact outflow from FIR 4 that contributes to the CO emission. When combining their results with those of Manoj et al. (2013), Kama et al. (2013) derived a total CO luminosity of $\sim 0.4 L_{\odot}$. If we assume an $L_{\text {bol }}$ value of $37 L_{\odot}$, the CO luminosity amounts to $1 \%$ of the total energy output. However, it is not clear whether the protostar associated with FIR 4 is responsible for the entire $\mathrm{CO}$ emission. Using interferometric observations, Shimajiri et al. (2008) argued that the high velocity CO emission toward FIR 4 is due to the outflow from FIR 3 colliding with the FIR 4 clump. In their interpretation, the morphology of the FIR 4 core arises from the interaction with the FIR 3 outflow (also see López-Sepulcre et al. 2013). In this case, the far-IR CO lines may have a significant contribution from the shock driven by FIR 3 .

\subsection{The Molecular Clump Associated with OMC-2 FIR 4}

Besides the offset between the $8-70 \mu \mathrm{m}$ peak and the peak at $4.5 \mu \mathrm{m}$, Figure 9 also shows the offset between the 70 and $160 \mu \mathrm{m}$ peaks mentioned in Section 2. The $160 \mu \mathrm{m}$ peak also roughly coincides with the peak position in our APEX data and those reported at $2 \mathrm{~mm}$ (López-Sepulcre et al. 2013) and $3.3 \mathrm{~mm}$ (Shimajiri et al. 2008; see also Table 4). Thus, the more extended emission at $\lambda \geqslant 160 \mu \mathrm{m}$ probably probes a dense clump of dust and gas heated externally. This scenario is similar to the Bok globule studied by Stutz et al. (2010) containing a protostar and a starless core, with the emission from the latter source starting to be noticeable at $\lambda>100 \mu \mathrm{m}$ and becoming comparable to the emission from the protostar in the submillimeter.

To examine the properties of this molecular core, we integrated the flux in a $20^{\prime \prime}$ aperture centered on the core position, using an annulus from $30^{\prime \prime}$ to $40^{\prime \prime}$ to subtract out the more extended emission. For the core position, we used the average centroid position from the 160, 350 and $870 \mu \mathrm{m}$ maps $(\alpha(\mathrm{J} 2000)=$ $5^{\mathrm{h}} 35^{\mathrm{m}} 26^{\mathrm{s}} .88$ and $\left.\delta(\mathrm{J} 2000)=-05^{\circ} 9^{\prime} 57^{\prime \prime} .8\right)$. After applying the aperture corrections for a point source (which primarily account for flux scattered to large angles in the $160 \mu \mathrm{m}$ data), we fit a modified blackbody to the $160-870 \mu \mathrm{m}$ photometry using the same opacity law from Ormel et al. (2011) as for the HOPS model grid and our models. The resulting fit gives a temperature of $22 \mathrm{~K}$, a mass of $27.3 M_{\odot}$, and an overall luminosity of $137 L_{\odot}$. This is consistent with our earlier model that combined a protostellar model and a modified blackbody fit to our standard fluxes from aperture photometry (with aperture radii $<20^{\prime \prime}$; thus, our earlier fit yielded a somewhat smaller temperature of $18.5 \mathrm{~K}$ ).

Our analysis shows that the FIR 4 core is massive with a mass commensurate with that of a high-mass star. Although the possibility of higher temperatures due to the heating by the protostar may affect the measurement, the localized mass should be within a factor of two of the observed mass and therefore would still be in excess of $10 M_{\odot}$. Our mass is consistent with the masses determined from the interferometer data by LópezSepulcre et al. (2013) for a range of assumed temperatures. Li et al. (2013) derived a core mass of $13 M_{\odot}$ based on $\mathrm{NH}_{3}$ data; they inferred that the FIR 4 core was just massive enough to be in virial equilibrium and thus gravitationally bound. The placement of the protostar near the edge of the submillimeter clump is consistent with the claim of López-Sepulcre et al. (2013) and Shimajiri et al. (2008) that the submillimeter core has fragmented and is forming multiple objects, with the observed protostar to be the first object to form with a high enough luminosity to be detected. We also note that due to the large reservoir of gas mass associated with the protostar, although the observed protostar currently appears to have a modest luminosity, it may continue to grow in mass and luminosity as it draws from the core. Hence, the final mass of the protostar, and whether it may form a low-mass star or intermediate-mass star, is highly uncertain.

Given that the long-wavelength $(\lambda \geqslant 100 \mu \mathrm{m})$ emission from FIR 4 is dominated by the massive core that is mostly heated externally, the protostar itself is probably best described by our models that fit the mid-IR data and the PSF photometry at $70 \mu \mathrm{m}$ and resulted in $L_{\text {tot }}$ values ranging from 14 to $23 L_{\odot}$ (see Section 4 and Table 3). Also, given the absence of a wide outflow from FIR 4, SED models that require a high total luminosity and large cavity opening angles seem more unrealistic. A young, spatially compact outflow would not have had sufficient time to carve a large cavity within the envelope. Among the lowluminosity models (Models 4, 6, 8, and 10), those with a larger inclination angle $\left(>60^{\circ}\right)$ have wider cavities and somewhat larger $L_{\text {tot }}$ values (such that sufficient mid-infrared photons can still reach the observer despite the high inclination), so we favor the two models with $i=49^{\circ}$ and luminosities of 14-15 $L_{\odot}$. In addition, the models with lower inclination angles (which typically require larger envelope densities) seem to result in deeper silicate absorption features and lower near-infrared fluxes, which better matches the observations.

\section{CONCLUSIONS}

OMC-2 FIR 4 is an intriguing protostar whose nature has been debated in the literature; it is likely deeply embedded and thus in an early evolutionary stage, but its properties, like luminosity and envelope mass, were poorly determined. We clearly detect protostellar emission at $\lambda \leqslant 70 \mu \mathrm{m}$, but at longer wavelengths the larger molecular core dominates the emission. We present the most complete analysis to date of this object. Using data from the Spitzer, Herschel, and APEX telescopes, we derive new values for the bolometric luminosity of OMC-2 FIR 4 and estimate some of its envelope properties from model fits. Some ambiguities on the detailed nature remain due to the deeply embedded state of the protostar. Our main conclusions are as follows:

1. We construct the SED of OMC-2 FIR 4 with photometry at $8,24,37.1,70,100,160,350$, and $870 \mu \mathrm{m}$, and spectroscopy from 5 to $37 \mu \mathrm{m}$. Thus, the SED is wellsampled, in particular at wavelengths where the emission peaks. We obtain more accurate photometry of the protostar and its envelope by choosing smaller apertures $\left(\sim 10^{\prime \prime}\right)$ in the $70-870 \mu \mathrm{m}$ range than were previously adopted. However, we note an offset of $\sim 3^{\prime \prime}$ in the emission peak for $\lambda \leqslant 70 \mu \mathrm{m}$ and $\lambda \geqslant 160 \mu \mathrm{m}$, which suggests that at long wavelengths we actually probe a clump of externally heated dust and thus 
even our fluxes at 160,350 , and $870 \mu \mathrm{m}$ could overestimate the envelope emission.

2. The bolometric luminosity of OMC-2 FIR 4 ranges from $37 L_{\odot}$ to $100 L_{\odot}$, depending on which values are adopted for the far-IR and submillimeter photometry. Given that the extended emission surrounding this object at long wavelengths $(\gtrsim 70 \mu \mathrm{m})$ may be dominated by a cold, externally heated clump, the $L_{\text {bol }}$ value most closely describing the protostar is likely $37 L_{\odot}$.

3. Models that include a protostar surrounded by a disk and envelope with outflow cavities fit the SED well. These models yield different best-fit parameters depending on which photometry values are adopted and which model assumptions are made. Assuming a single protostar with an infalling envelope, we estimate that the envelope density is relatively high $\left(\rho_{1} \sim 10^{-13}-10^{-12} \mathrm{~g} \mathrm{~cm}^{-3}\right.$ or $\rho_{1000} \sim$ $3 \times 10^{-18}-3 \times 10^{-17} \mathrm{~g} \mathrm{~cm}^{-3}$ ), both for models with polynomial-shaped and streamline-shaped cavities.

4. The SED can also be fit by combining a protostellar model that considers fluxes between 8 and $70 \mu \mathrm{m}$ and a clump of externally heated dust that fits the longerwavelength emission. In this model the luminosity is dominated by the clump, and the total luminosity of the protostar alone amounts to $\sim 15-25 L_{\odot}$ (with corresponding $L_{\text {bol }}$ values of $\left.12-14 L_{\odot}\right)$. The envelope density is still high $\left(\rho_{1}\right.$ close to $10^{-13} \mathrm{~g} \mathrm{~cm}^{-3}$ or $\rho_{1000}$ close to $3 \times$ $10^{-18} \mathrm{~g} \mathrm{~cm}^{-3}$ ), suggesting an early evolutionary state for the protostar (Stage 0). Given the significant contribution of the molecular clump to the long-wavelength emission, the protostar is probably best described by this model.

5. We find that the position of OMC-2 FIR 4 measured in our IRAC $4.5 \mu \mathrm{m}$ image is offset with respect to the position measured at $8-70 \mu \mathrm{m}$, but matches that of the radio continuum source detected at $3.6 \mathrm{~cm}$ by Reipurth et al. (1999). Both can be interpreted as emission from shocked gas in an outflow. Furthermore, there is evidence in favor of an outflow from far-IR spectra (Manoj et al. 2013; Kama et al. 2013) in the form of velocity profiles, temperatures, and densities derived from CO lines, although they may contain a contribution from an outflow driven by the nearby protostar OMC-2 FIR 3. These data support the idea that FIR 4 is indeed a protostar, driving a compact outflow. In addition, the centimeter flux is consistent with that observed in outflows from other protostars with luminosities $<100 L_{\odot}$ (Anglada 1995).

6. Using fluxes measured in a $20^{\prime \prime}$ aperture centered on the clump position (i.e., the position of the peak flux at $\lambda \geqslant$ $160 \mu \mathrm{m}$ ) and applying a modified blackbody fit, we estimate a temperature of $22 \mathrm{~K}$ and a mass of $27 M_{\odot}$ for the clump. This clump could form more protostars, and OMC-2 FIR 4, which lies near its edge, might be the first one formed, but is probably still growing in mass and luminosity. Thus, we agree with the suggestion of Shimajiri et al. (2008) and López-Sepulcre et al. (2013) that the molecular core of OMC-2 FIR 4 likely fragmented, with one of these fragments currently containing a protostar. However, we find that the data are best explained by a $<100 L_{\odot}$ protostar and not an intermediate-mass, luminous $\left(\sim 1000 L_{\odot}\right)$ young star as proposed by Crimier et al. (2009) and LópezSepulcre et al. (2013). Although the protostar currently has a modest luminosity, the final stellar mass it will obtain is difficult to predict considering that it is embedded in a core with a total mass of $27 M_{\odot}$.
Only long-wavelength observations at high spatial resolution, such as the VLA and ALMA can provide, will allow us to better understand this object. In particular, mapping the dust continuum and the outflows at resolutions $\lesssim 1^{\prime \prime}$ will constrain the envelope structure, including the properties of the cavity and inclination angle. This in turn will settle the question about this object's luminosity. Overall, OMC-2 FIR 4 will further our understanding of the star formation process in complex environments such as OMC 2.

This work is based on observations made with the Spitzer Space Telescope, which is operated by the Jet Propulsion Laboratory (JPL), California Institute of Technology (Caltech), under a contract with NASA; it is also based on observations made with the Herschel Space Observatory, a European Space Agency Cornerstone Mission with significant participation by NASA. The Herschel spacecraft was designed, built, tested, and launched under a contract to ESA managed by the Herschel/ Planck Project team by an industrial consortium under the overall responsibility of the prime contractor Thales Alenia Space (Cannes), and including Astrium (Friedrichshafen) responsible for the payload module and for system testing at spacecraft level, Thales Alenia Space (Turin) responsible for the service module, and Astrium (Toulouse) responsible for the telescope, with in excess of a hundred subcontractors. We also include data from the Atacama Pathfinder Experiment, a collaboration between the Max-Planck Institut für Radioastronomie, the European Southern Observatory, and the Onsala Space Observatory. Support for this work was provided by NASA through awards issued by JPL/Caltech. The work of A.M.S. was supported by the Deutsche Forschungsgemeinschaft priority program 1573 ("Physics of the Interstellar Medium"). M.O. acknowledges support from MICINN (Spain) AYA2008-06189C03-01 and AYA2011-30228-C03-01 grants (co-funded with FEDER funds).

\section{APPENDIX}

Models assuming different cavity shapes (polynomial versus streamline) can result in widely different model parameters. Here we explore the effect of the cavity shape on the SED. In Figures 10 and 11 we show Model 4 from Section 4 with different cavity opening angles and at two inclination angles $\left(49^{\circ}\right.$ in Figure 10 and $70^{\circ}$ in Figure 11; all other model parameters are unchanged).

At both inclination angles, the differences between models with a streamline-shaped cavity and those with a polynomialshaped cavity become pronounced when the cavity opening angle is fairly large, $\gtrsim 25^{\circ}$. Then the polynomial-shaped cavity, which evacuates more material, allows more shorter-wavelength photons to reach the observer; the silicate absorption feature and the mid-IR SED slope become shallower. At an inclination angle of $49^{\circ}$, there is a noticeable difference between the two types of cavity already at $\theta=15^{\circ}$. As the cavity opening angle reaches $45^{\circ}$, the stellar and disk emission are unobscured with a polynomial-shaped cavity, while a streamline-shaped cavity still leaves sufficient envelope dust along the line of sight to cause a deep silicate absorption feature and a steeply rising SED in the mid-IR. Only the models with $\theta=5^{\circ}$ agree well irrespective of cavity shape. This is also reflected in our modeling results from Section 4, where the best-fit model parameters of Model 4 (which assumed a polynomial-shaped cavity and had $\theta=5^{\circ}$ ) are 

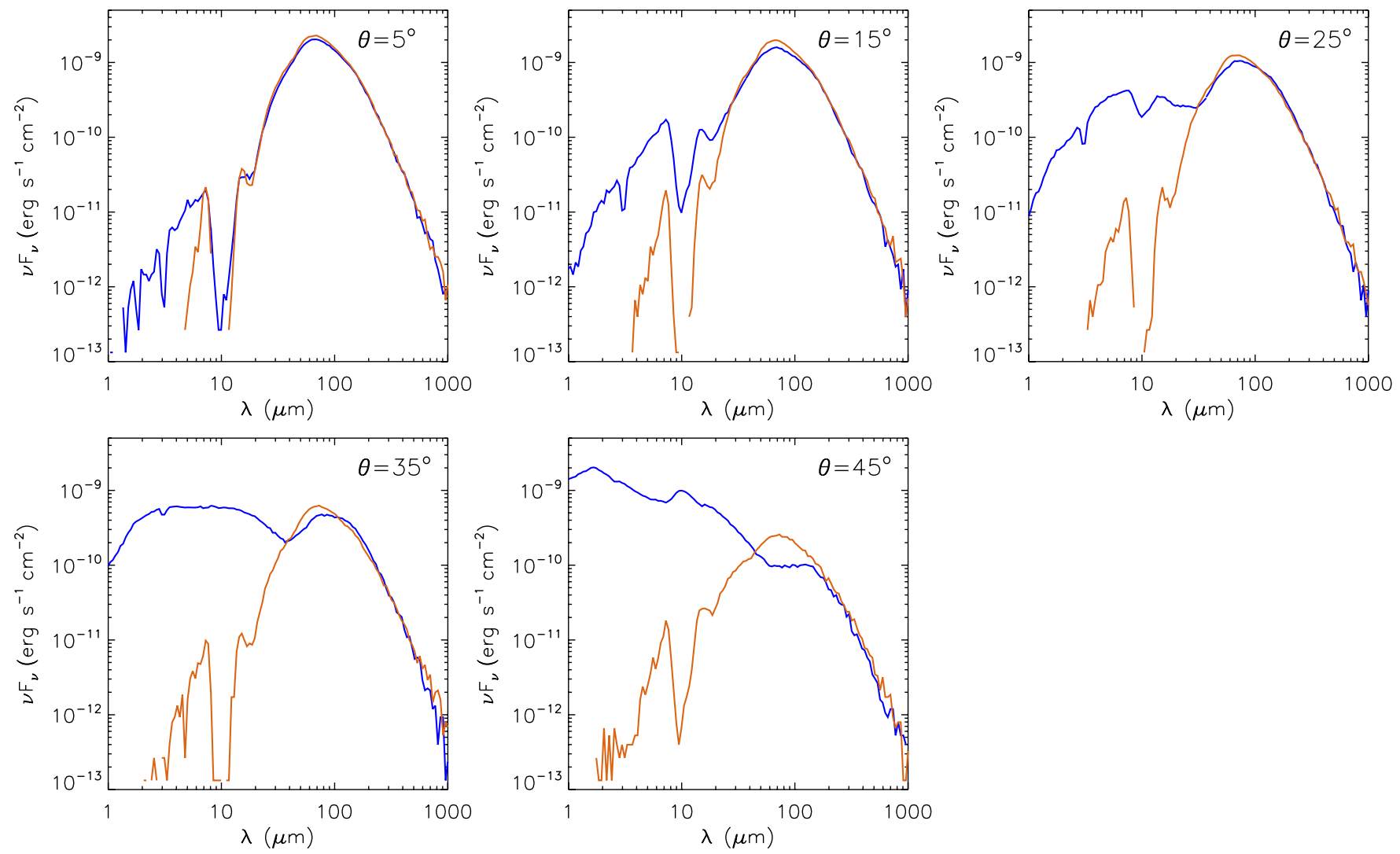

Figure 10. Model 4 from Section 4, calculated with the same parameters except for the cavity: in the five different panels, the same model is shown with five different cavity opening angles (see label inside each panel), and in each panel for two different cavity shapes (blue: polynomial-shaped cavity with exponent 1.5; orange: streamline-shaped cavity). Note that Model 4 has an inclination angle of $49^{\circ}$, and the best fit to OMC-2 FIR 4 has $\theta=5^{\circ}$.

(A color version of this figure is available in the online journal.)
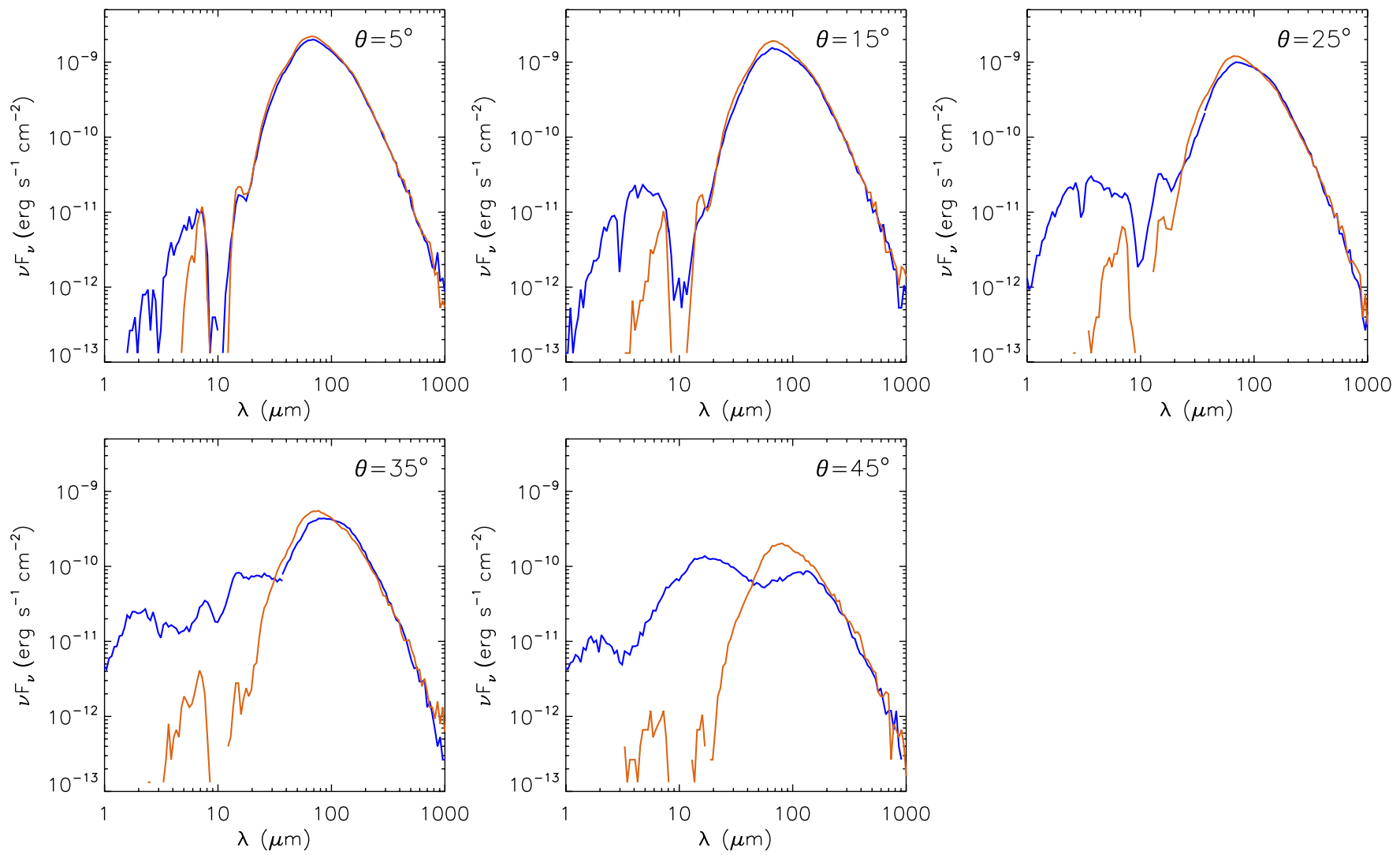

Figure 11. Same as in Figure 10, but the models are shown for an inclination angle of $70^{\circ}$.

(A color version of this figure is available in the online journal.) 
very similar to those of Model 8 (which assumed a streamlineshaped cavity and also had $\theta=5^{\circ}$ ).

\section{REFERENCES}

Adams, J. D., Herter, T. L., Osorio, M., et al. 2012, ApJL, 749, L24 Ali, B., \& DePoy, D. L. 1995, AJ, 109, 709

André, P., Men'shchikov, A., Bontemps, S., et al. 2010, A\&A, 518, L102

André, P., Ward-Thompson, D., \& Barsony, M. 1993, ApJ, 406, 122

Anglada, G. 1995, Rev. Mex. AA Ser. Conf., 1, 67

Anglada, G. 1996, in ASP Conf. Ser. 93, Radio Emission from the Stars and the Sun, ed. A. R. Taylor \& J. M. Paredes (San Francisco, CA: ASP), 3 Carpenter, J. M. 2000, AJ, 120, 3139

Chen, H., Myers, P. C., Ladd, E. F., \& Wood, D. O. S. 1995, ApJ, 445, 377

Chini, R., Reipurth, B., Ward-Thompson, D., et al. 1997, ApJL, 474, L135

Crimier, N., Ceccarelli, C., Lefloch, B., \& Faure, A. 2009, A\&A, 506, 1229

D’Alessio, P., Calvet, N., Hartmann, L., et al. 1999, ApJ, 527, 893

D'Alessio, P., Calvet, N., Hartmann, L., et al. 2006, ApJ, 638, 314

Diolaiti, E., Bendinelli, O., Bonaccini, D., et al. 2000, A\&AS, 147, 335

Fazio, G. G., Hora, J. L., Allen, L. E., et al. 2004, ApJS, 154, 10

Fischer, W. J., Megeath, S. T., Ali, B., et al. 2010, A\&A, 518, L122

Fischer, W. J., Megeath, S. T., Tobin, J. J., et al. 2012, ApJ, 756, 99

Furlan, E., McClure, M., Calvet, N., et al. 2008, ApJS, 176, 184

Gatley, I., Becklin, E. E., Matthews, K., et al. 1974, ApJL, 191, L121

Hartmann, L., Calvet, N., \& Boss, A. 1996, ApJ, 464, 387

Herter, T. L., Adams, J. D., De Buizer, J. M., et al. 2012, ApJL, 749, L18

Hirota, T., Bushimata, T., Choi, Y. K., et al. 2007, PASJ, 59, 897

Houck, J. R., Roellig, T. L., van Cleve, J., et al. 2004, ApJS, 154, 18

Johnson, J. J., Gehrz, R. D., Jones, T. J., et al. 1990, AJ, 100, 518

Johnstone, D., \& Bally, J. 1999, ApJL, 510, L49

Johnstone, D., Boonman, A. M. S., \& van Dishoeck, E. F. 2003, A\&A, 412, 157

Jones, T. J., Mergen, J., Odewahn, S., et al. 1994, AJ, 107, 2120

Kama, M., López-Sepulcre, A., Dominik, C., et al. 2013, A\&A, 556, A57

Kenyon, S. J., Calvet, N., \& Hartmann, L. 1993, ApJ, 414, 676
Li, D., Kauffmann, J., Zhang, Q., \& Chen, W. 2013, ApJL, 768, L5

Lis, D. C., Serabyn, E., \& Keene, J. 1998, ApJ, 509, 299

López-Sepulcre, A., Taquet, V., Sánchez-Monge, Á., et al. 2013, A\&A, 556, A62

Manoj, P., Watson, D. M., Neufeld, D. A., et al. 2013, ApJ, 763, 83

Mathis, J. S. 1990, ARA\&A, 28, 37

McClure, M. 2009, ApJL, 693, L81

Megeath, S. T., Gutermuth, R., Muzerolle, J., et al. 2012, AJ, 144, 192

Menten, K. M., Reid, M. J., Forbrich, J., \& Brunthaler, A. 2007, A\&A, 474, 515

Mezger, P. G., Wink, J. E., \& Zylka, R. 1990, A\&A, 228, 95

Nielbock, M., Chini, R., \& Müller, S. A. H. 2003, A\&A, 408, 245

Ormel, C. W., Min, M., Tielens, A. G. G. M., et al. 2011, A\&A, 532, A43

Peterson, D. E., \& Megeath, S. T. 2008, in Handbook of Star Forming Regions, Vol. 1, ed. B. Reipurth (Provo, UT: ASP Monograph Publications), 590

Pilbratt, G. L., Riedinger, J. R., Passvogel, T., et al. 2010, A\&A, 518, L1

Poglitsch, A., Waelkens, C., Geis, N., et al. 2010, A\&A, 518, L2

Rayner, J., McLean, I., McCaughrean, M., \& Aspin, C. 1989, MNRAS, 241, 469

Reipurth, B., Rodríguez, L. F., \& Chini, R. 1999, AJ, 118, 983

Rieke, G. H., Young, E. T., Engelbracht, C. W., et al. 2004, ApJS, 154, 25

Robitaille, T. P., Whitney, B. A., Indebetouw, R., et al. 2006, ApJS, 167, 256

Shimajiri, Y., Takahashi, S., Takakuwa, S., et al. 2008, ApJ, 683, 255

Siringo, G., Kreysa, E., De Breuck, C., et al. 2010, Msngr, 139, 20

Siringo, G., Kreysa, E., Kovács, A., et al. 2009, A\&A, 497, 945

Smith, M. D., \& Rosen, A. 2005, MNRAS, 357, 1370

Stanke, T., Stutz, A. M., Tobin, J. J., et al. 2010, A\&A, 518, L94

Stutz, A. M., Launhardt, R., Linz, H., et al. 2010, A\&A, 518, L87

Stutz, A. M., Tobin, J. J., Stanke, T., et al. 2013, ApJ, 767, 36

Terebey, S., Shu, F. H., \& Cassen, P. 1984, ApJ, 286, 529

Tsujimoto, M., Koyama, K., Kobayashi, N., et al. 2003, AJ, 125, 1537

Werner, M. W., Roellig, T. L., Low, F. J., et al. 2004, ApJS, 154, 1

Whitney, B. A., Wood, K., Bjorkman, J. E., \& Cohen, M. 2003b, ApJ, 598, 1079

Whitney, B. A., Wood, K., Bjorkman, J. E., \& Wolff, M. J. 2003a, ApJ, 591, 1049

Young, E. T., Becklin, E. E., Marcum, P. M., et al. 2012, ApJL, 749, L17 